\title{
Comparative transcriptome analysis of the wild-type model apomict Hieracium praealtum and its loss of parthenogenesis (lop) mutant
}

\author{
Sophia Bräuning ${ }^{1,2}$, Andrew Catanach ${ }^{3}$, Janice M. Lord ${ }^{2}$, Ross Bicknell ${ }^{3}$ and Richard C. Macknight ${ }^{1^{*}}$ (D)
}

\begin{abstract}
Background: Asexual seed formation (apomixis) has been observed in diverse plant families but is rare in crop plants. The generation of apomictic crops would revolutionize agriculture, as clonal seed production provides a low cost and efficient way to produce hybrid seed. Hieracium (Asteraceae) is a model system for studying the molecular components of gametophytic apomixis (asexual seed reproduction).

Results: In this study, a reference transcriptome was produced from apomictic Hieracium undergoing the key apomictic events of apomeiosis, parthenogenesis and autonomous endosperm development. In addition, transcriptome sequences from pre-pollination and post-pollination stages were generated from a loss of parthenogenesis (lop) mutant accession that exhibits loss of parthenogenesis and autonomous endosperm development. The transcriptome is composed of 147,632 contigs, $50 \%$ of which were annotated with orthologous genes and their probable function. The transcriptome was used to identify transcripts differentially expressed during apomictic and pollination dependent (lop) seed development. Gene Ontology enrichment analysis of differentially expressed transcripts showed that an important difference between apomictic and pollination dependent seed development was the expression of genes relating to epigenetic gene regulation. Genes that mark key developmental stages, i.e. aposporous embryo sac development and seed development, were also identified through their enhanced expression at those stages.
\end{abstract}

Conclusion: The production of a comprehensive floral reference transcriptome for Hieracium provides a valuable resource for research into the molecular basis of apomixis and the identification of the genes underlying the LOP locus.

Keywords: Apomixis, Parthenogenesis, Asexual, Hieracium, RNA sequencing, Transcriptome

\section{Background}

Apomixis is a type of asexual plant reproduction that bypasses meiosis and fertilization to result in viable seeds that are maternal clones [1]. It is observed in around 400 plant species belonging to 40 families, however very few of these plants are crop species [2]. Introducing the apomixis trait into commercial hybrid seeds would allow fixing hybrid vigor [2]. Because of this agricultural application the ability to transfer or engineer

\footnotetext{
* Correspondence: richard.macknight@otago.ac.nz

'Department of Biochemistry, University of Otago, 710 Cumberland St,

Dunedin 9016, New Zealand

Full list of author information is available at the end of the article
}

apomixis into crop species is the ultimate goal of many studies of apomixis. As a result, many model plants have been developed to study apomixis [3-10].

Gametophytic apomixis is just one of a range of apomictic developmental pathways. It is characterized by the formation of unreduced embryo sacs either from somatic nucellar cells called aposporous initials (AI) (apospory) or from the Megaspore Mother Cell (MMC) (diplospory), and the subsequent development of the embryo from the unreduced egg cell through parthenogenesis. In some cases, the central cell proliferates autonomously to give rise to the endosperm, however usually it requires fertilization by one sperm cell

(c) The Author(s). 2018 Open Access This article is distributed under the terms of the Creative Commons Attribution 4.0 International License (http://creativecommons.org/licenses/by/4.0/), which permits unrestricted use, distribution, and 
(pseudogamy) [1]. Most apomicts are pseudogamous, with the exception of a few autonomous apomicts (aposporous), mainly in Asteraceae [11]. The genus Hieracium L. (Asteraceae) contains sexual and apomictic species that are aposporous (subgenus Pilosella) and diplosporous (subgenus Hieracium) [12]. Recently, subgenus Pilosella and subgenus Hieracium were treated as separate genera, with the aposporous species belonging to genus Pilosella and the diplosporous species belonging to the genus Hieracium [13]. In this study we still refer to the aposporous group as Hieracium subgenus Pilosella.

Hieracium subgenus Pilosella has been developed as a model system to investigate the molecular components of gametophytic apomixis [14]. Various studies have utilized this model to further our understanding of the cytological and molecular events in this group. For instance, the floral staging in relation to gametogenesis, embryogenesis and seed maturation events in sexual and apomictic members of this group was described by Koltunow et al. [9]. Key regulatory processes during seed development were shown to be similar in sexual and apomictic species of Hieracium subgenus Pilosella; the differences occurred temporally and spatially [15]. Additionally, the genomic regions conferring apomeiosis and parthenogenesis in $H$. caespitosum, also in subgenus Pilosella, were identified through a deletion mapping study [16]. These are independent loci termed LOSS OF APOMEIOSIS (LOA) and LOSS OF PARTHENOGENESIS ( $L O P)$, respectively. $L O A$ is involved in the formation of aposporous initials and their subsequent development to make aposporous embryo sacs. Therefore, plants with deleted regions in this locus employ meiosis to produce reduced embryo sacs. These mutants, however, retain the ability of the egg cell and central cell to undergo spontaneous proliferation to give rise to the embryo and endosperm respectively. $L O P$ is responsible for parthenogenesis. The deletion of this region is associated with mutants that retain the ability to produce aposporous embryo sacs but require fertilization in order for the embryo and endosperm to develop. These mutants carry the recessive allele (lop). Mutants with loss of function in both loci were also found and these mutants employ the sexual pathway to produce seeds [16].

This model system offers unique opportunities to study apomixis. For example, the lack of near identical apomictic and sexual lines has hindered the discovery of transcriptionally active apomixis-linked genes in Paspalum simplex [17]. The Hieracium model system overcomes this challenge as it encompasses natural apomicts as well as mutants that either completely or partially lost the ability to reproduce asexually. The lack of genomic and transcriptomic reference material for Hieracium is another challenge for high throughput analysis of gene expression. We aimed to produce a reference floral transcriptome of Hieracium, which will be a useful tool for comparative analysis of gene expression in apomictic, sexual and mutant Hieracium.

We carried out a comparative transcriptomics study of an apomictic Hieracium accession (R35) and its pollination dependent (lop) mutant. This allowed us to compare gene expression during parthenogenesis in the wild type to that which occurs during fertilization-induced embryo development in the mutant accession. This reference transcriptome will be useful in advancing our knowledge about regulatory networks and pathways involved in apomixis. It will also aid the efforts being made to map the genes found in the LOP region of Hieracium.

\section{Results \\ Determining capitulum stages of R35, lop138 and A36 that are undergoing embryo development}

To profile the expression of genes involved in autonomous seed development, embryo screening was initially used to determine the capitulum stages to be sampled. The wild-type apomictic line, R35, contained mature embryo sacs by stage 6 and had commenced embryogenesis by stage 7 . The lop 138 mutant contained mature embryo sacs by stage 6 but was not able to proceed through embryogenesis unless cross-pollinated. The pollinator line, A36, despite being aposporous, took longer to produce mature embryo sacs and initiated embryo development later than R35. These observations allowed the identification of capitulum stages to be sampled in order to produce the transcriptomic data. To investigate transcriptional changes occurring during parthenogenesis and fertilization induced embryo developments, stages before embryogenesis and after embryogenesis for R35 and lop 138 were selected. An "after embryogenesis" stage was also selected for A36 (Table 1).

\section{RNA isolation from the reproductive tissues sampled}

To obtain sufficient RNA for analysis, the reproductive tissue from different staged capitula needed to be manually dissected then pooled. As both the dissection and pooling steps can lead to RNA degradation in the tissue, various solutions were trialed to find a suitable tissue storage and dissection media during the tissue collection process. From these trials it was found that RNAase free water could be used as a dissection medium. Replicated sampling was carried out from the stages listed in Table 1. Multiple ovules and ovaries (50-93) were pooled to isolate $\sim 1.4-4.5 \mu \mathrm{g}$ of total RNA (Additional file 1: Table S1). 
Table 1 Information of developmental events in sampled capitula stages of R35, lop138 and A36

\begin{tabular}{|c|c|c|c|c|c|}
\hline Species & Accession Id & Parthenogenesis & Sample code & Capitulum stage & Developmental events ${ }^{\#}$ \\
\hline \multirow[t]{16}{*}{ Hieracium praealtum } & \multirow[t]{12}{*}{ R35 } & \multirow[t]{12}{*}{ Capable } & Ovary_3 & 3 & Tetrads of megaspores present, Als differentiating \\
\hline & & & Ovary_4 & 4 & $\begin{array}{l}\text { Meiosis suppression, meiotic tetrads degenerating } \\
\text { and Als enlarging }\end{array}$ \\
\hline & & & Ovary_5 & 5 & Embryo sacs develop from Als through mitosis \\
\hline & & & Ovary_6 & 6 & $\begin{array}{l}\text { Mature embryo sacs developed. Some undergo } \\
\text { spontaneous embryogenesis. }\end{array}$ \\
\hline & & & Ovary_7 & 7 & $\begin{array}{l}\text { Embryo and endosperm development occur in } \\
\text { many ovules. }\end{array}$ \\
\hline & & & Ovary_8 & 8 & Ovules contain early to globular embryos \\
\hline & & & Ovary_9 & 9 & Ovules contain early to globular embryos \\
\hline & & & Ovary10 & 10 & $\begin{array}{l}\text { Senescence begins. Ovules contain globular to late } \\
\text { globular embryos. }\end{array}$ \\
\hline & & & Wt_E1 & \multirow[t]{2}{*}{5} & \multirow[t]{2}{*}{ Embryo sacs develop from Als through mitosis } \\
\hline & & & Wt_E2 & & \\
\hline & & & Wt_L1 & \multirow[t]{2}{*}{7} & \multirow{2}{*}{$\begin{array}{l}\text { Embryo and endosperm development occur in } \\
\text { many ovules. }\end{array}$} \\
\hline & & & Wt_L2 & & \\
\hline & \multirow[t]{4}{*}{ lop 138} & \multirow[t]{4}{*}{ Not capable } & Lop_E1 & \multirow[t]{2}{*}{9 (not pollinated) } & \multirow[t]{2}{*}{ Mature embryo sacs containing quiescent egg cell } \\
\hline & & & Lop_E2 & & \\
\hline & & & Lop_L1 & \multirow[t]{2}{*}{12 (after pollination) } & \multirow[t]{2}{*}{ Ovules contain globular to heart? embryos } \\
\hline & & & Lop_L2 & & \\
\hline \multirow[t]{2}{*}{ H. aurantiacum } & \multirow[t]{2}{*}{ A36 } & \multirow[t]{2}{*}{ Capable } & Pol_L1 & \multirow[t]{2}{*}{10} & \multirow[t]{2}{*}{ Ovules contain globular to heart stage embryos } \\
\hline & & & Pol_L2 & & \\
\hline
\end{tabular}

\# Description of developmental events for R35 and A36 was based on [9, 51] and observations from the current study and [16] were used for lop138

\section{De novo assembly}

Sequencing of the prepared cDNA libraries on Illumina's HiSeq 2000 produced a total of 791,095,876 good quality reads. Trinity assembly yielded a total of 179,195 transcripts belonging to 85,000 components (loci). For each library, $75-81 \%$ of reads were mapped back to the reference transcriptome, therefore the transcriptome is representative of all libraries and the sub-sampling of reads to produce a reference transcriptome was deemed to have been effective.

The N50 and average transcript length of the transcriptome were around 500 bp. BUSCO assessment revealed that the transcriptome was incomplete and had some redundancy. The transcriptome was further processed to remove chimeric and redundant transcripts, which resulted in an assembly containing 147,632 transcripts belonging to 74,424 loci. This reduced the number of duplicated BUSCOs by $10 \%$. This reference transcriptome has been deposited at the Shotgun Assembly project DDBJ/EMBL/GenBank under the accession number GEEH00000000.

\section{Transcriptome annotation}

To annotate the proteins potentially encoded in these transcripts, BLASTX searches were performed against two protein databases with an e-value cut-off of $1 \mathrm{e}-4$.
The first database contained all NCBI-NR plant protein sequences and BLASTX against it returned similarities for 35,373 of Hieracium transcripts (47.5\%). The second database was Swiss-Prot, which is manually curated and often supported via experimental or literature evidence. BLASTX against this database identified similarity with 25,377 (34\%) of the transcripts. After consolidating the results, a total of 35,436 transcripts (48\%) were identified with similarity to proteins in these databases.

The e-value distribution of these BLASTX hits is shown in Fig. 1a and revealed that $30 \%$ showed very high similarity, having an e-value lower than 1e-50. Figure 1b displays the distribution of percent similarity of the BLASTX results and indicates that $87 \%$ of the matches have over $25 \%$ percent identity. $52 \%$ of the genes had no match in the databases. As expected, the percentage of genes with similarity increases with gene length (Fig. 1c). For example, 32\% of the genes whose length ranged from 201 to 400 bp had database hits, while $90 \%$ of those longer than $1000 \mathrm{bp}$ had hits (Fig. 1c). Thus, the reason why more than half of the transcripts lack similarity is likely to be because they do not have a protein coding sequence due to their short length. However, included in this group are transcripts encoding novel proteins and polyadenylated non-coding RNAs. 


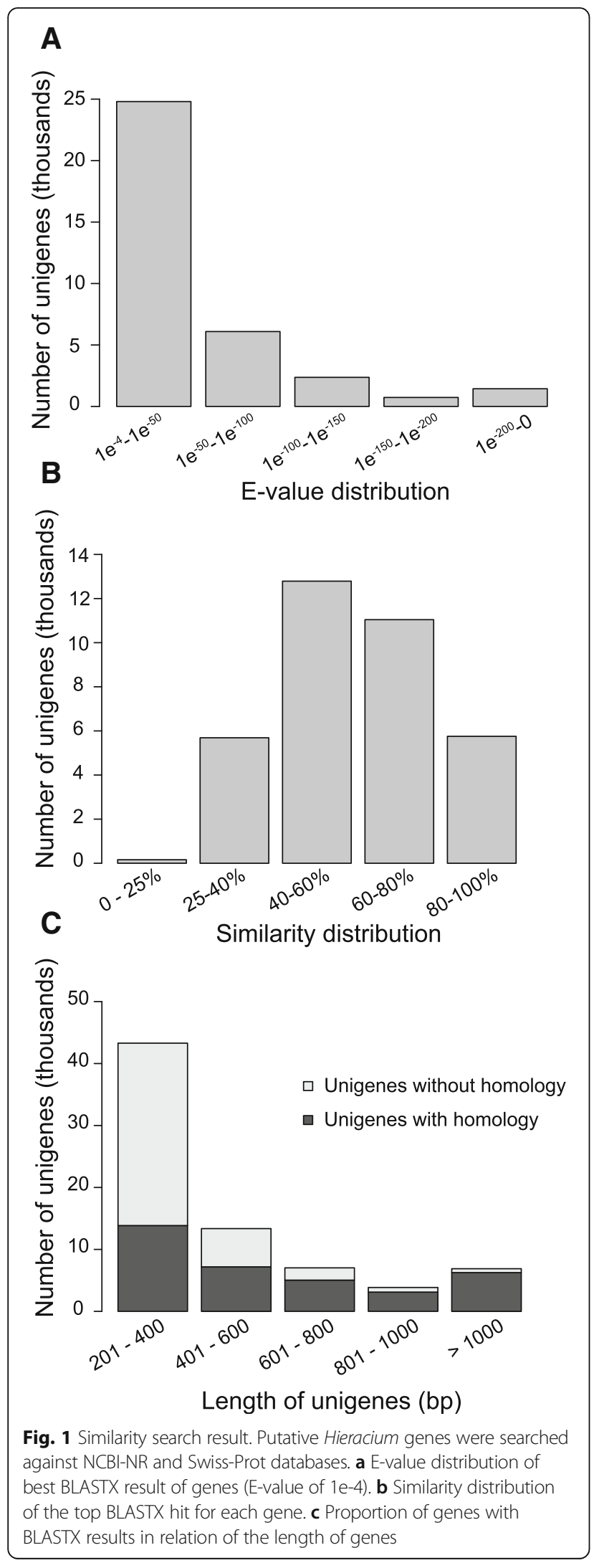

Transcript abundance across the libraries was estimated by counting reads aligned to each transcript from each library. The raw read counts were normalized and scaled using the TPM and UPC methods of normalization.

\section{Identification of differentially expressed (DE) transcripts}

Capitula of Hieracium carry multiple florets that mature asynchronously. As a result, samples from consecutive capitulum stages were expected to represent overlapping developmental stages. To determine the degree of overlap in expressed transcripts between stages, Spearman's correlation was applied between each consecutive pair of samples. The expression profiles of all samples were positively correlated with that of the prior developmental stage, with the majority having a correlation coefficient above 0.7 (Additional file 1: Table S2). Based on this observation, it was decided that samples produced from consecutive capitulum stages could be combined in order to carry out comparative analysis. Four main comparisons were made to identify expression changes occurring in association with apomictic development and in association with fertilization-induced seed development (Table 2). Additional file 1: Table S3 records the number of DE transcripts identified in these analyses. The results of the comparative analyses conducted using DESeq are presented in Additional files 2, 3, 4, 5.

Four comparisons were performed using DE transcripts to discover enriched GO terms (Table 2). Comparison 1 and 2 aimed to identify genes and processes associated with autonomous seed development by comparing samples with mature embryo sacs to samples where spontaneous embryogenesis has occurred (Additional files 2 and 3). GO enrichment analysis of transcripts corresponding to initiation of autonomous seed development (comparison 1) resulted in enrichment of 86 GO terms (Additional file 1: Figure S2). Most of the enriched terms were pertinent to growth and organ morphogenesis, such as regulation of cell growth, regulation of meristem growth, stomatal complex morphogenesis and regulation of organ morphogenesis. GO enrichment analysis of transcripts corresponding to maturing embryos and endosperms in R35 (comparison 2), resulted in 299 enriched GO terms that are summarized in (Additional file 1: Figure S3). In addition to cell and organ growth related terms, this set contained GO terms of embryo and post-embryonic development; such as cotyledon vascular tissue pattern formation, lateral root development, inflorescence morphogenesis, seed coat development, mucilage extrusion from seed coat, seed germination and maintenance of seed dormancy.

Comparison 3 aimed to discover functions enriched due to pollination-induced seed development in the parthenogenesis-incapable accession (lop138). This resulted in 384 enriched GO terms (Additional file 4) 


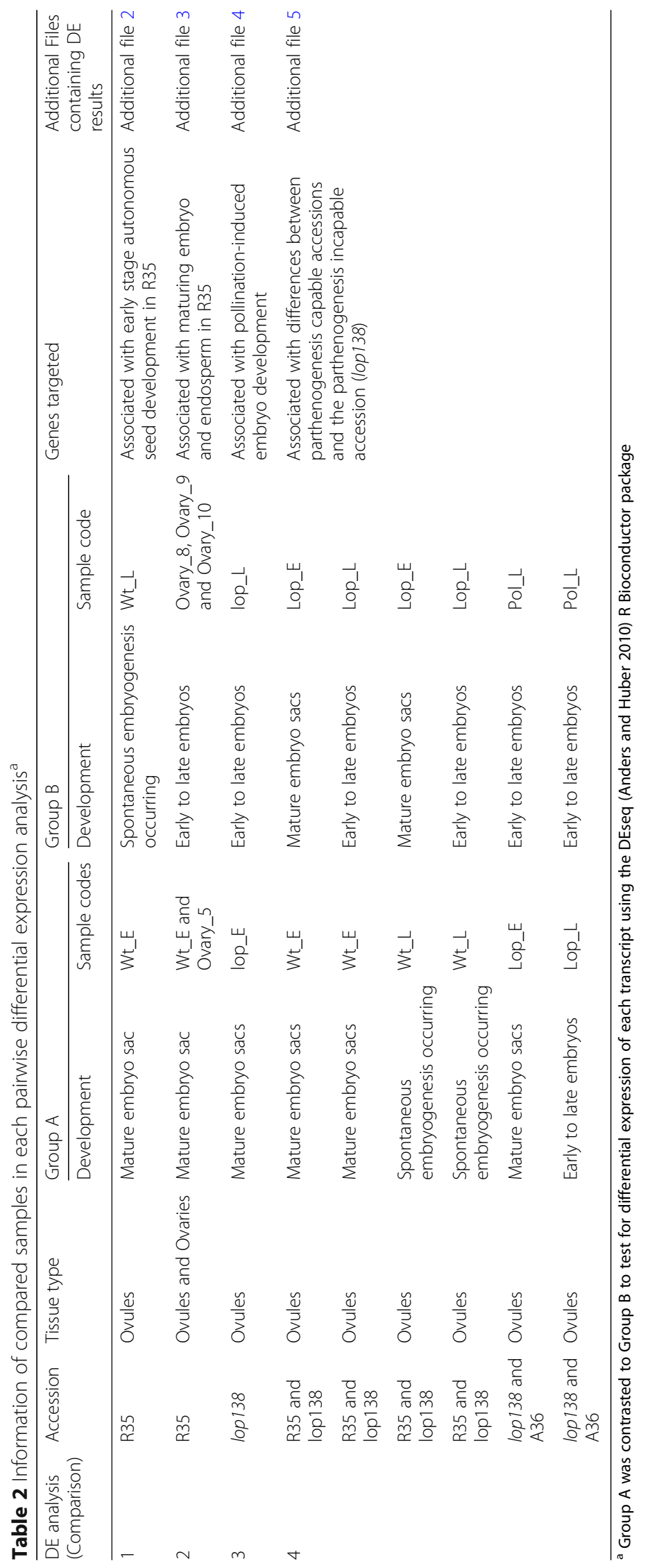


belonging to the broad GO term categories (Additional file 1: Figure S4). Most terms enriched in this set were also enriched in DE sets of R35 (Additional file 1: Figures S2 \& S3). However, in the 'Reproduction' category, GO terms associated with sexual reproduction were enriched for lop 138 only, which indicated that in the absence of the $L O P$ locus the aposporous embryo sac was functionally equivalent to a sexual embryo sac. The specific 'sexual reproduction' terms were; regulation of double fertilization forming a zygote and endosperm, regulation of induction of conjugation upon nitrogen starvation, pre-meiotic DNA replication and megasporogenesis. In addition, the GO term 'epigenetic regulation of gene expression' was enriched for lop138 but not for R35.

Comparison 4 aimed to discover functions associated with differences between the parthenogenesis-capable accessions and the parthenogenesis-incapable accession (lop138). A total of 16,530 sequences that were DE in at least one pairwise comparison (lop138 vs. apomictic accessions) were analyzed to identify enriched GO terms (Table 2). This resulted in the enrichment of 518 GO terms (Additional file 5), belonging to the $\mathrm{GO}$ categories detailed in (Additional file 1: Figure S5). The enriched GO categories were similar to the GO categories enriched in the DE sets of R35 (resulting from comparison 2, Additional file 3) and lop138 (resulting from comparison 3, Additional file 4).

The GO analyses were performed using all the transcripts in the reference datasets that had GO annotations (Additional file 6). A limitation of the GO analysis is that only $48 \%$ of the transcriptome was annotated. Therefore, there are a large number of DE transcripts without GO annotation (some of which have BLAST hits but no associated GO annotation) which could not be included in the GO analyses. These transcripts are listed in Additional file 7 and (Additional file 1: Table S4) provides a summary of the number of DE transcripts without GO terms in the different categories.

In summary, all DE sets resulting from the four comparisons shared enriched GO terms. The main differences between the DE sets were that terms associated with epigenetic regulation of gene expression and sexual reproduction were enriched for lop138 but not for R35. This observation indicated that a lot of transcripts involved in epigenetic regulation and other sexual reproduction processes were differentially expressed during pollinationinduced seed development in lop138 but not in R35, which undergoes autonomous seed development.

\section{Global expression patterns of differentially expressed transcripts \\ Epigenetic regulation}

Hierarchical clustering was used to explore global expression changes of DE transcripts annotated with epigenetic regulation of gene expression (Fig. 2; Additional file 8). The clustering analysis highlighted that most of the transcripts that were up-regulated during embryo development in lop138 (Fig. 2) were associated with DNA methylation and chromatin silencing functions (Additional file 8). Many of the transcripts associated with epigenetic regulation were up-regulated in lop 138, showing with a high expression at early megagametogenesis stages of R35 (Ovary_3, Ovary_4) and continuing to show some level of expression in the ovules at stage 5 (Wt_E) and stage 7 (Wt_L) (Fig. 2). This suggests that during autonomous seed development in R35, genes putatively involved in DNA methylation and chromatin silencing were expressed but did not show dramatic changes in expression in response to embryo and endosperm development. This could relate to the absence of a paternal genome in the developing seeds of R35, which may require less restructuring of the epigenome state in the central cell and early endosperm. In sexual plants such as maize, rice and Arabidopsis, demethylation of genomic DNA occurs prior to fertilization of the central cell [18-21]. Demethylation allows transcription of transposable elements (TEs) and the production of small interfering RNA (siRNA) that can be used as mobile elements to recognize and silence TEs in the embryo post-fertilization. As the deletion of the $L O P$ locus restores sexuality in the aposporous embryo sac of lop138, global demethylation may have occurred in the unpollinated ovules of lop138. Following pollination, TEs in the embryo of lop138 may be epigenetically silenced leading to up-regulation of many putative genes involved in epigenetic regulation.

Relatively few transcripts associated with epigenetic regulation were up-regulated in the pre-pollination sample of lop138. In R35, these transcripts showed high expression in both pre-embryo (Wt_E) and post-embryo (Wt_L) samples (group 1, Fig. 2). Many of these transcripts, belonging to group 1 in Fig. 2, encode putative orthologs of argonaute 4 (AGO4). Argonaute (AGO) proteins are core components of small RNA-induced silencing pathways; they can act in the post-transcriptional gene silencing (PTGS) pathway and the transcriptional gene silencing (TGS) pathway.

\section{Heterochrony}

The results from the differential expression and GO term enrichment analysis did not identify mechanisms that were unique to the parthenogenesis-capable wild type R35. This suggested that the differences between apomictic and lop138 seed development were likely to be spatio-temporal. Many studies propose apomixis to be the result of deregulation of the sexual reproduction pathway; therefore, during apomixis genes and regulatory mechanisms of sexual reproduction are employed 
in a heterochronic fashion $[14,15,22-24]$. To explore heterochrony in Hieracium, global expression patterns of the differentially expressed transcripts with the GO term category 'reproduction' were investigated (Fig. 3). Within this category, the GO term 'megasporogenesis' was enriched due to the expression of transcripts predicted to code proteins similar to the transcriptional regulator STERILE APETALA (SAP), squamosa promoter-binding-like protein 8 (SPL8) and protein TORNADO 2 (TRN2) (Fig. 3). In Arabidopsis SPL8, $S A P$ and TRN2 have regulatory roles that are important for initiation of female gametogenesis and other developmental processes [25]. Therefore, if putative orthologs of SPL8, SAP and TRN2 perform similar tasks in Hieracium as in Arabidopsis, these genes can be expected to be more active during stages involving female gametogenesis, which in wild type Hieracium is complete around stage 6 (Table 3 ).
The reproduction associated GO term 'pre-meiotic DNA replication' was enriched due to putative orthologs of DNA replication licensing factor $m c m 2-7$ (MCM2-7) complex and replication factor A protein 1 (RFA1) (Table 3). The MCM2-7 complex proteins plays a role that ensures replication occurs once per cell cycle [26]. RFA1 is conserved in all eukaryotes and is required for DNA replication, repair and recombination [27]. Based on their roles during cell division, the expression of the putative $M C M 2-7$ complex and RFA1 can be expected to increase during stages undergoing cellular proliferation. In lop138, cell division stops after the embryo sac matures, therefore expression of genes involved in cell division can be expected to increase in the post-pollination stage as cell division resumes during embryogenesis. In R35, the mature embryo sac proceeds through spontaneous embryogenesis; therefore, expression of genes involved in cell division may not differ dramatically between

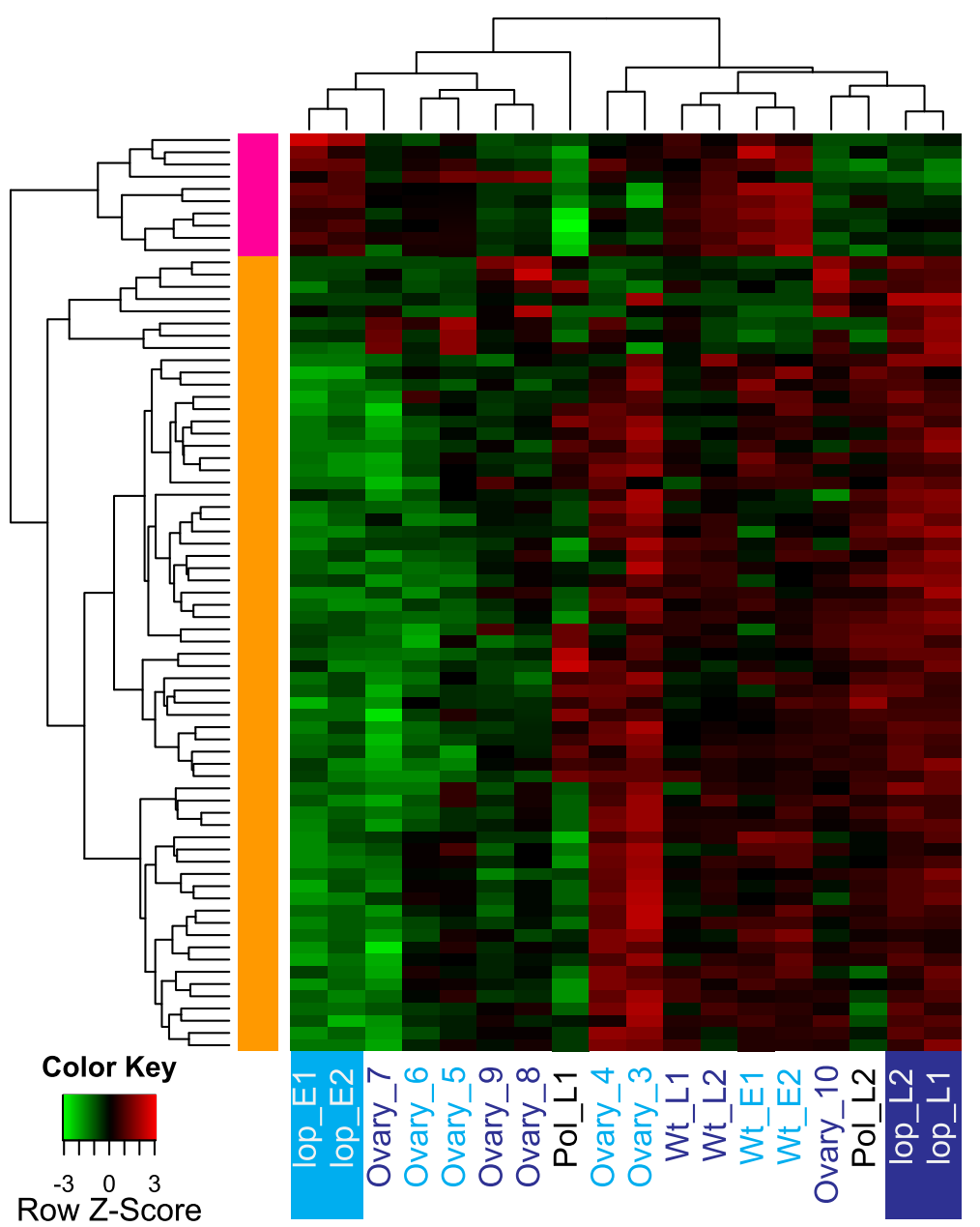

Fig. 2 Expression patterns of DE transcripts associated with epigenetic regulation. Hierarchically clustered expression heatmap with rows represent each transcript and relative expression (low in green and high in red) in the samples as indicated. Pre-embryo stages are indicated in light blue and post-embryo stages in dark blue text; the lop mutant pre-pollination (Iop E1, E2) and post pollination (Iop L1 and L2) are also highlighted) 
the mature embryo sac and the post-embryo developmental stage. Putative orthologs of MCM2 and MCM6 were identified as up-regulated in lop138 post-pollination but not in R35 post-embryo samples (Fig. 3; Additional file 1: Tables S3, S4 and S5). Although not DE in R35 post-embryo samples relative to pre-embryo samples, putative MCM2 and MCM6 exhibited relatively high expression in stage 3 and 4 samples of R35 (Fig. 3). In R35, putative RFA1 expression was high during stages undergoing female gamete development (3-5) and early embryo development (stages 6-7). In lop 138, putative RFA1 expression did not change during embryo development (Fig. 3).

The reproduction associated GO term 'regulation of induction of conjugation upon nitrogen starvation' was enriched due to transcripts predicted to encode proteins with similarity to cell differentiation protein RCD1, which is involved in the onset of sexual reproduction through its differentiation controlling function in yeast [28]. Four transcripts predicted to encode RCD1 were up-regulated in the post-pollination sample of lop 138 and also showed high expression in the post-embryo sample of A36 (Fig. 3). Compared to pre-embryo stages (3-5) of R35, three of these putative $R C D 1$ orthologs also showed increased expression in post-embryo stages (7-10) of R35 (Fig. 3). The GO term 'regulation of double fertilization forming a zygote and endosperm' was enriched because of transcripts that are likely to encode proteins similar to egg cell-secreted (EC1) proteins of Arabidopsis. In Arabidopsis, five functionally redundant
EC1-like genes (EC1.1, EC1.2, EC1.3, EC1.4 and EC1.5) were specifically expressed in the egg cell prior to fertilization and regulated gamete interaction during double fertilization [29]. Based on EC1's function and expression patterns in Arabidopsis, putative EC1 was expected to be active in lop138, which employs double fertilization during seed development. While six putative $E C 1$ orthologs were identified as DE in lop138, EC1 orthologs were not identified as DE in R35 (Additional files 2, 3, 4,5). Although not DE in R35, putative EC1 orthologs showed some level of expression in both pre-embryo (Wt_E) and post-embryo (Wt_L) ovule samples as well as in the post-embryo A36 sample (Fig. 3). Up-regulation of one of the putative EC1 orthologs in post-pollination sample of lop138 may indicate that some egg cells had not yet initiated embryogenesis.

For transcripts that were DE in R35 relative to lop138 samples, the 'reproduction' category contained the GO terms 'cotyledon vascular tissue pattern formation', 'maintenance of seed dormancy', 'mucilage extrusion from seed coat' and 'reproductive structure development'. Figure 4 shows the expression patterns of transcripts associated with these terms. Transcripts associated with floral structure development were mostly up-regulated in the R35 pre-embryo samples and putatively encode $A B C$ transporter $B$ family member 19 (AB19B), peroxidase 12 (PER12), piriformospora indicainsensitive protein 2 (PII-2), somatic embryogenesis receptor kinase 1 (SERK 1), regulatory protein NPR5 (NPR5) and lipoxygenase 3 (LOX3) (Fig. 4). Many of

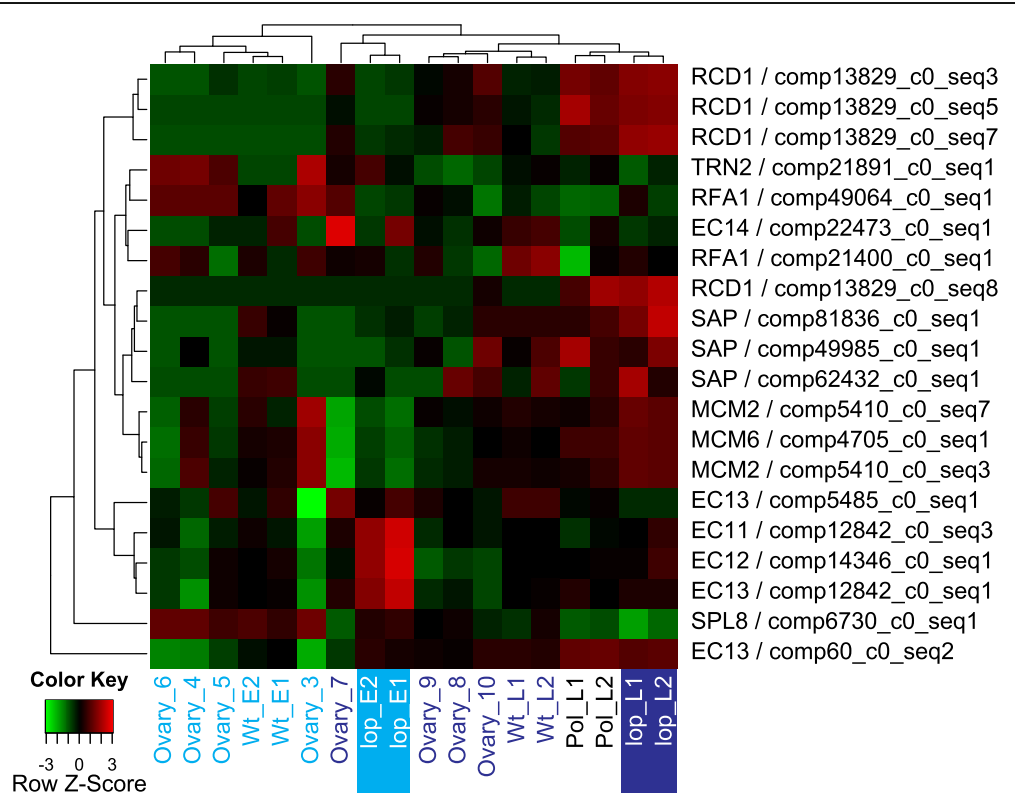

Fig. 3 Expression pattern of DE transcripts (Iop138 pre- vs post-pollination) associated with the GO category 'reproduction'. Hierarchically clustered expression heatmap with rows represent each transcript (labeled by the putative protein and the transcript ID) and relative expression (low in green and high in red) in the samples as indicated. Pre-embryo stages are indicated in light blue and post-embryo stages in dark blue text; the lop mutant pre-pollination (Iop E1, E2) and post pollination (Iop L1 and L2) are also highlighted) 
these proteins play a role in plant development through their involvement in auxin transport (AB19B) [30], response to biotic and abiotic stress (perioxidases) [31], mediating symbiotic relationship of fungus and host to promote growth and seed production (PII-2) [32], promoting leaf and floral meristem fate and determinacy through positive regulation of adaxial-abaxial polarity genes such as YAB1 and YAB3 (NPR5) [33, 34] and involvement of LOXs in plant growth, pest resistance, senescence or responses to wounding [35, 36]. Most notably SERK genes code for a transmembrane protein involved in signal transduction and have been associated with somatic embryogenesis and apomixis and function in plant response to biotic and abiotic stimuli in a number of plant species [37]. Therefore up-regulation of putative SERK1 transcripts in pre-embryo samples of R35 may be an indication that this gene plays a role in inducing parthenogenesis in R35. Transcripts associated with mucilage extrusion from the seed coat were putative orthologs of subtilisin-like protease (SUBL). More transcripts with similarity to SUBL were up-regulated in the pre-embryo samples of R35 (Fig. 4).

Putative orthologs of $\mathrm{ABC}$ transporter G family member 11 (AB11G) and $A B C$ transporter $G$ family member
14 (AB14G) were associated with cotyledon vascular tissue pattern formation. These transcripts were up-regulated in the post-embryo samples of R35 (Fig. 4). Transcripts associated with maintenance of seed dormancy were putative orthologs of TRANSPARENT TESTA 12 (TT12) and were up-regulated in the post-embryo samples of R35 (Fig. 4). The expression patterns observed for transcripts likely encoding AB11G, AB14G and TT12 fit with their associated function in cotyledon tissue formation and seed maturation as seed development progressed in R35.

\section{Embryo patterning genes and polar auxin transport}

During early embryogenesis, asymmetrical division in the zygote establishes the apical-basal axis of the embryo. While the basal cell transports the growth regulator hormone auxin, the apical cell responds to auxin and this apical-basal gradient of auxin triggers the specification of apical embryo structures. Auxin carriers such as PIN-FORMED1 (PIN1) and their polar localization play an important role in the specification of apical-basal pattern formation of early embryo and root formation. The polar localization of auxin carriers is dependent on directed vesicle trafficking, which is regulated by the ARF guanine-nucleotide exchange factor GNOM [38]. A

Table 3 Summary of expression pattern of transcripts differentially expressed in lop 138, putatively encoding for proteins involved in reproduction

\begin{tabular}{|c|c|c|c|c|}
\hline GO term & Proteins & Transcript ID & Stage showing high expression in R35 & $\begin{array}{l}\text { Stage showing high } \\
\text { expression in lop138 }\end{array}$ \\
\hline \multirow[t]{5}{*}{ Megasporogenesis } & SAP & Comp81836_c0_seq1 & $\begin{array}{l}\text { Pre-embryo (stage 5) and post-embryo } \\
\text { (stages } 7 \text { and 10) }\end{array}$ & Post-pollination \\
\hline & SAP & Comp49985_c0_seq1 & Post-embryo (stages 7 and 10) & Post-pollination \\
\hline & SAP & Comp62432_c0_seq1 & $\begin{array}{l}\text { Pre-embryo (stage 5) and post-embryo } \\
\text { (stages } 7,8 \text { and 10) }\end{array}$ & Post-pollination \\
\hline & SPL8 & Comp6730_c0_seq1 & Pre-embryo (stages 3-6) & Pre-pollination \\
\hline & TRN2 & Comp21891_c0_seq1 & Pre-embryo (stages 3-6) & Pre-pollination \\
\hline \multirow[t]{4}{*}{ Pre-meiotic DNA replication } & MCM2 & Comp5410_c0_seq7 & Pre-embryo (stages 3 and 4) & Post-pollination \\
\hline & MCM2 & Comp5410_c0_seq3 & Pre-embryo (stages 3 and 4) & Post-pollination \\
\hline & MCM6 & Comp4705_c0_seq1 & Pre-embryo (stages 3 and 4) & Post-pollination \\
\hline & RAF1 & Comp49064_c0_seq1 & Pre-embryo and post-embryo (stages 3-7) & Unchanged \\
\hline \multirow[t]{4}{*}{$\begin{array}{l}\text { Regulation of induction of conjugation } \\
\text { upon nitrogen starvation }\end{array}$} & RCD1 & Comp13829_c0_seq3 & Post-embryo (stages 7-10) (three sequences) & $\begin{array}{l}\text { Post-pollination } \\
\text { (four sequences) }\end{array}$ \\
\hline & RCD1 & Comp13829_c0_seq5 & Post-embryo (stages 7-10) & Post-pollination \\
\hline & RCD1 & Comp13829_c0_seq7 & Post-embryo (stages 7-10) & Post-pollination \\
\hline & RCD1 & Comp13829_c0_seq8 & unchanged & Post-pollination \\
\hline \multirow{6}{*}{$\begin{array}{l}\text { Regulation of double fertilization } \\
\text { forming a zygote and endosperm }\end{array}$} & EC1.4 & Comp22473_c0_seq1 & Pre-embryo (stage 5) and Post-embryo (stage 7) & Pre-pollination \\
\hline & EC1.3 & Comp5485_c0_seq1 & Pre-embryo (stage 5) and Post-embryo (stage 7) & Pre-pollination \\
\hline & EC1.1 & Comp12842_c0_seq3 & Pre-embryo (stage 5) and Post-embryo (stage 7) & Pre-pollination \\
\hline & EC1.2 & Comp14346_c0_seq1 & Pre-embryo (stage 5) & Pre-pollination \\
\hline & EC1.3 & Comp12842_c0_seq1 & Pre-embryo (stage 5) and Post-embryo (stage 7) & Pre-pollination \\
\hline & EC1.3 & Comp60_c0_seq2 & Post-embryo (stages 8-10) & Post-pollination \\
\hline
\end{tabular}


Hieracium transcript putatively encoding GNOM was up-regulated in the pre-embryo stages of R35 (Fig. 5). Many transcripts associated with auxin transport were also up-regulated in the pre-embryo stages of R35. These were $\mathrm{AB} 19 \mathrm{~B}$, serine/threonine-protein kinases WAG1 and WAG2, MAX1 and PIN proteins. Most of the PIN proteins were also up-regulated in the post-pollination sample of lop138 (Fig. 5). AB19B is involved in auxin transport and together with PIN proteins, establishes the polar auxin concentration gradient [30]. WAG1 and WAG2 are also involved in the regulation of auxin signaling through enhancing PIN-mediated polar auxin transport [39]. MAX genes are proposed to inhibit axillary bud growth by affecting PIN-mediated auxin transport in the stem [40]. Up-regulation of transcripts involved in auxin transport in the pre-embryo stages of R35 indicates that the phytohormone auxin and its concentration gradient in ovaries of Hieracium may be an important cue for induction of autonomous embryo development.

WUSCHEL (WUS) regulates stem cell formation and maintenance [41] and in Arabidopsis is first expressed at the 16-cell stage of embryogenesis in cells that will form the shoot apical meristem, or SAM [42]. A plant specific homeodomain transcription factor, WUSCHEL-RELATED
HOMEOBOX9 (WOX9), promotes stem-cell fate by positively regulating the expression of WUSCHEL (WUS) in the SAM [43]. In R35, putative WUS homolog expression was high in pre-embryo stages (Fig. 5), which could indicate that an earlier induction of WUS in apomictic ovaries of Hieracium promotes stem cell identity and maintenance, leading to precocious somatic embryogenesis. The putative homolog of WOX9 also showed up-regulation in pre-embryo and post-embryo ovaries of R35, which further indicates that stem cell specification may begin during early ovary development prior to the morphological observation of the apomictic embryo. Other embryo developmental patterning genes, such as SCARECROW (SCR) [44] and YABBY genes [45] were also identified (Fig. 5).

\section{Endosperm development}

While the endosperm develops autonomously in R35, it requires fertilization in lop138. Transcripts predicted to encode proteins with similarity to proteins that play critical roles in endosperm development in other plants such as members of the PcG complex (FIS1/MEA, FIS2, FIS3/FIE, RBR and MSI1), were investigated to find out if they differentially express in R35 or lop138. Expressions of three members of FIS complex (FIE, RBR and

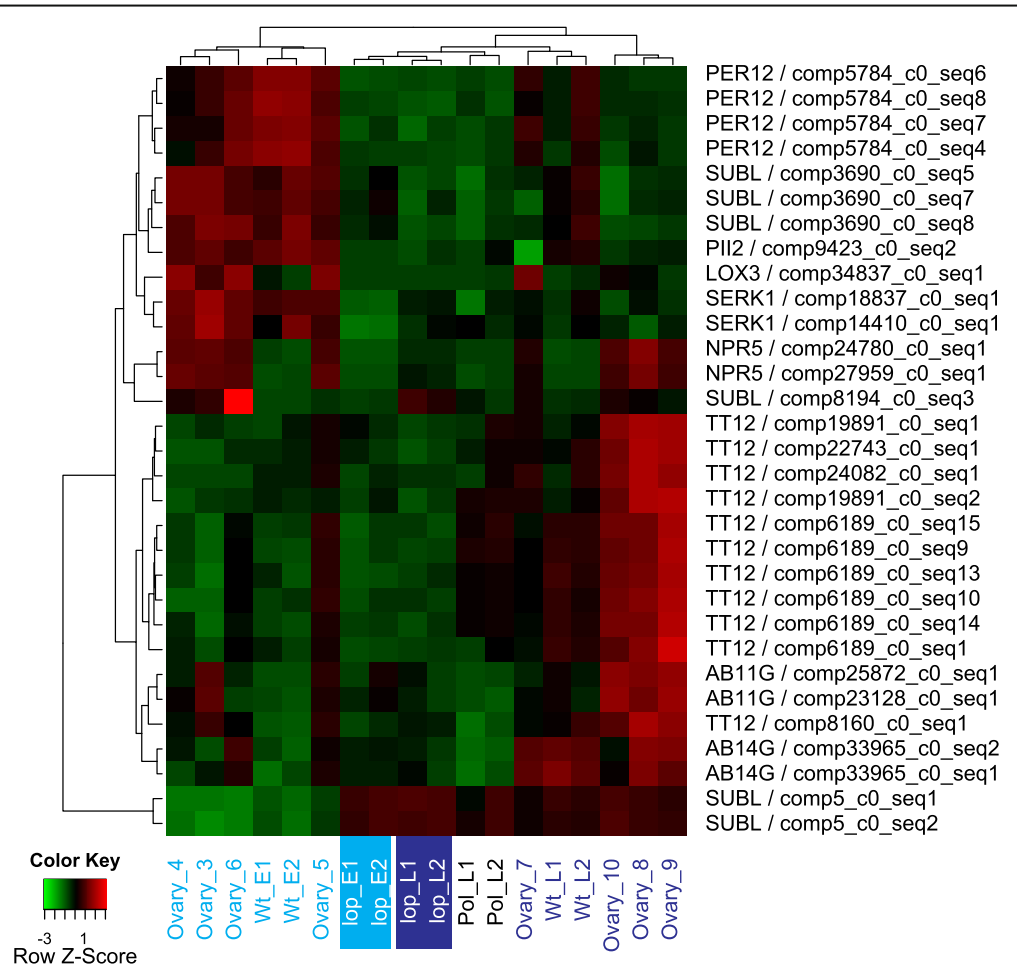

Fig. 4 Expression patterns of DE transcripts (Iop138 vs WT) associated with the GO category 'reproduction'. Hierarchically clustered expression heatmap with rows represent each transcript (labeled by the putative protein and the transcript ID) and relative expression (low in green and high in red) in the samples as indicated. Pre-embryo stages are indicated in light blue and post-embryo stages in dark blue text; the lop mutant pre-pollination (Iop E1, E2) and post pollination (Iop L1 and L2) are also highlighted) 
MSI1) were supported by the high percent similarities between the respective Arabidopsis protein sequences and the predicted Hieracium peptides (above 70\% identity). However, only three putative MSI1 orthologs were up-regulated during embryo development in lop138. There was no evidence of expression of MEA and FIS2 in Hieracium, however expression of SWINGER and $C L F$ was supported. Table 4 lists sequences predicted to encode PcG proteins and their DE status.

In Arabidopsis, Agamous-like MADS-box proteins (AGL61, AGL62 and AGL80) are required for central cell and endosperm development and are exclusively expressed in the central cell and uncellularized endosperm [46-48]. To gain insight into the expression of genes with known domains of expression in Arabidopsis, all DE transcripts putatively encoding AGL61, AGL62 or AGL80 proteins (Additional file 1: Table S5) were heat mapped (Fig. 6). The majority of the transcripts showed high expression at post-embryo stages of lop138, R35 (stages 7-10) and A36, which reflects the syncytial phase of endosperm development.

\section{Genes showing enhanced expression during a developmental stage}

The transcriptome produced for this study included consecutive developmental stages, ranging from megagametogenesis to embryogenesis and seed maturation processes in the ovary (Ovary_3 - Ovary_10). The ovary data set is therefore ideal for two purposes; 1) to investigate unique processes occurring at each capitulum stage, which may lead to the identification of processes contributing to initiation and progression of apomixis in R35, and 2) to identify molecular markers of key developmental stages.

To identify developmental stage markers for Hieracium, the expression of each gene was represented by a UPC value of 0 (inactive) to 1 (active) [49]. To simplify the analysis, only the longest transcript of each component was chosen as the representative for that component (gene). The UPC values were plotted across the time points in order to select those that showed expression peaks in a capitulum stage ( 0.5 being the cutoff) (Fig. 7). While stage 3 showed the highest level of genes

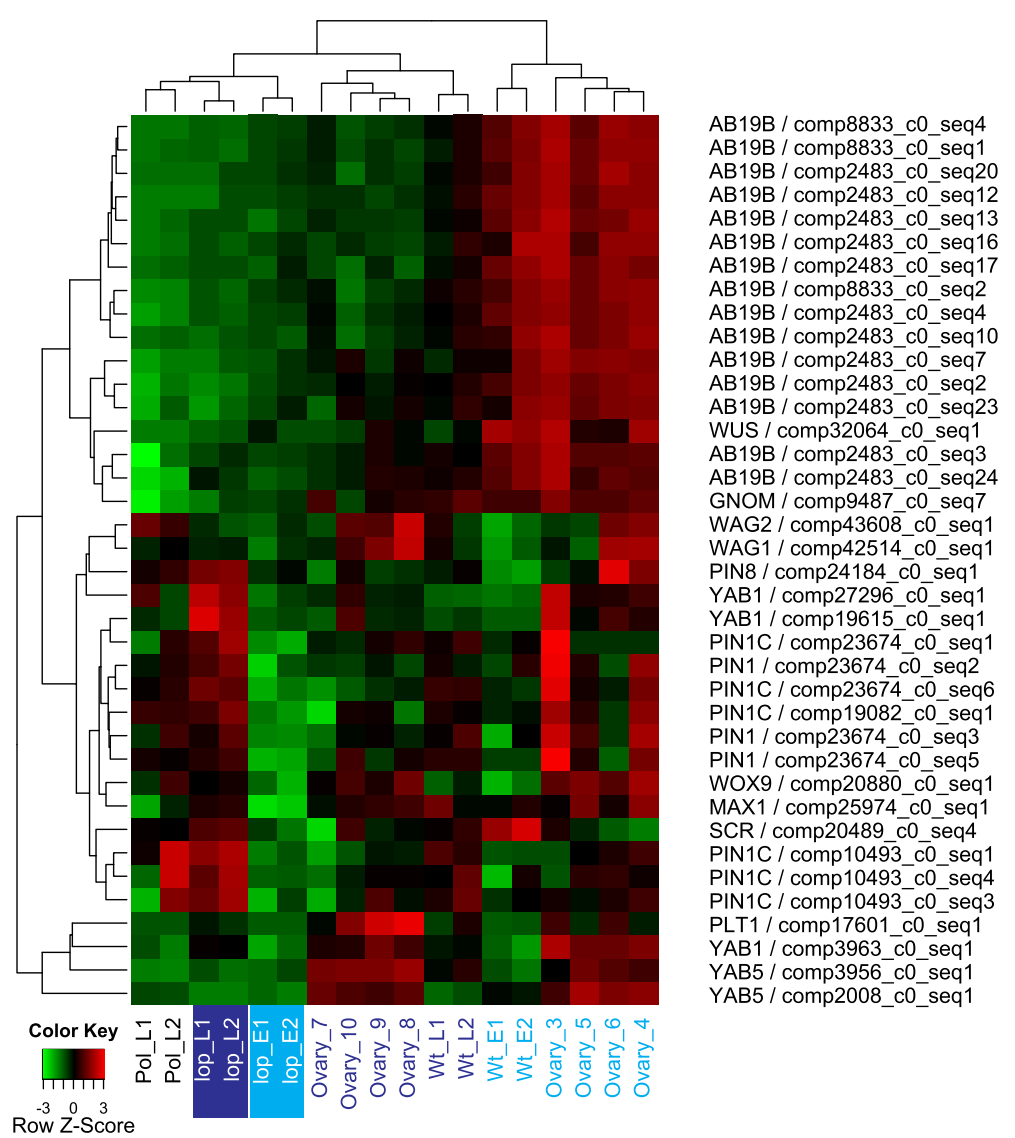

Fig. 5 Expression patterns of DE transcripts associated with auxin transport and embryo development. Hierarchically clustered expression heatmap with rows represent each transcript (labeled by the putative protein and the transcript ID) and relative expression (low in green and high in red) in the samples as indicated. Pre-embryo stages are indicated in light blue and post-embryo stages in dark blue text; the lop mutant pre-pollination (lop E1, E2) and post pollination (lop L1 and L2) are also highlighted) 
showing enhanced activity, stages 4, 5 and 10 exhibited low numbers of genes showing enhanced activity at these capitulum stages. Additional file 9 lists BLASTX results and read counts supporting expression of Hieracium genes showing stage-enhanced activity.

KOBAS 2.0 [50] was used to identify enriched pathways and GO terms in the gene sets showing stage-enhanced expression. Additional file 1: Table S6 presents enriched pathways for the stage-enhanced expression data sets. Capitulum stage-enhanced gene expression analysis of the ovary data set of R35 revealed that capitulum stage 3 , in which AIs are differentiating in the vicinity of the megaspore tetrads, was enriched in pathways involved in biosynthesis and degradation of IAA. IAA is the most common naturally occurring plant hormone of the auxin class and is involved in various aspects of plant growth and development (Frick and Strader, 2018). Capitulum stages in which the aposporous embryo sac develops and matures (stages 4 and 5), did not present many genes exhibiting enhanced expression during these stages, nor did they have enriched functions. Ovaries from capitula at stage 6 and 7, in which parthenogenesis is expected to initiate, had a relatively high number of genes with enhanced expression, however these gene sets did not result in enrichment of a reproduction pathway term (Additional file 1: Table S6).

\section{Developmental stage marker genes of Hieracium}

To identify reliable developmental stage markers for $H$. praealtum, a UPC value of 0.5 was used as a cutoff to identify genes active during specific developmental stages. Developmental stage markers were identified for: 1) stages undergoing aposporous embryo sac development in R35, i.e. stages 3 to 6 (Additional file $10 \mathrm{~A}$ ); 2) stages undergoing embryo development in R35 and lop138, i.e. stages 7-10 in R35 and post-pollination stage for lop138 (Additional file $10 \mathrm{~B}$ ); 3) Markers for apomictic R35; i.e. transcripts active at any stage in R35 but not active in one or both stages of lop138 (pre-pollination and post-pollination stages) (Additional file $10 \mathrm{C}$ ).

The transcripts were identified as potential markers for aposporous embryo sac development stages (Additional file 1: Table S7). Many of these were predicted to encode proteins playing a role in lipid transfer or lipid metabolism and three transcripts that showed similarity to the transcription factor genes, GATA18, IAA32 and bHLH61, were

Table 4 Transcripts predicted to encode Polycomb Group (PcG) proteins based on top BLASTX hits to Arabidopsis proteins

\begin{tabular}{|c|c|c|c|c|c|}
\hline $\begin{array}{l}\text { Transcript ID } \\
\text {. }\end{array}$ & Transcript length (bp) & Putative protein & $\%$ identity & e-value & Differentially expressed ${ }^{a}$ \\
\hline Comp8804_c0_seq1 & 1271 & FIE & 74 & 0 & NO \\
\hline Comp7474_c0_seq1 & 743 & RBR & 99.3 & $7 \mathrm{E}^{-99}$ & NO \\
\hline Comp7474_c0_seq4 & 281 & RBR & 100 & $1 E^{-57}$ & NO \\
\hline Comp7474_c0_seq5 & 266 & RBR & 98.86 & $2 \mathrm{E}^{-51}$ & NO \\
\hline Comp7474_c0_seq6 & 252 & RBR & 100 & $3 \mathrm{E}^{-47}$ & NO \\
\hline Comp7474_c0_seq7 & 222 & RBR & 100 & $5 E^{-46}$ & NO \\
\hline Comp22708_c0_seq1 & 945 & MSI1 & 62 & $8 \mathrm{E}^{-98}$ & $\begin{array}{l}\text { Up-regulated in lop138 post-pollination } \\
\text { relative to lop138 pre-pollination }\end{array}$ \\
\hline Comp22708_c0_seq2 & 339 & MSI1 & 74.11 & $9 \mathrm{E}^{-59}$ & $\begin{array}{l}\text { Up-regulated in lop138 post-pollination } \\
\text { relative to lop } 138 \text { pre-pollination }\end{array}$ \\
\hline Comp20599_c0_seq1 & 355 & MSI1 & 75 & $7 E^{-10}$ & $\begin{array}{l}\text { Up-regulated in lop138 post-pollination } \\
\text { relative to lop138 pre-pollination }\end{array}$ \\
\hline Comp2957_c0_seq1 & 1637 & MSI1 & 92.62 & 0 & NO \\
\hline Comp76669_c0_seq1 & 280 & MSI1 & 89.25 & $1 E^{-53}$ & NO \\
\hline Comp7074_c0_seq1 & 2434 & EZA1/SWINGER & 75.55 & 0 & NO \\
\hline Comp12352_c0_seq1 & 2455 & CLF & 68.98 & $8 \mathrm{E}^{-157}$ & NO \\
\hline Comp16067_c0_seq1 & 357 & CLF & 38.83 & $1 \mathrm{E}^{-10}$ & NO \\
\hline Comp16067_c0_seq6 & 233 & CLF & 64.47 & $3 \mathrm{E}^{-22}$ & NO \\
\hline Comp16067_c0_seq7 & 231 & CLF & 81.58 & $6 \mathrm{E}^{-20}$ & NO \\
\hline Comp16067_c0_seq8 & 218 & CLF & 53.85 & $2 E^{-14}$ & NO \\
\hline Comp16067_c0_seq9 & 216 & CLF & 60.32 & $1 E^{-13}$ & NO \\
\hline Comp16067_c0_seq10 & 204 & CLF & 76.47 & $1 E^{-31}$ & NO \\
\hline Comp16067_c0_seq11 & 202 & CLF & 56.52 & $7 \mathrm{E}^{-05}$ & NO \\
\hline
\end{tabular}

${ }^{a}$ The 'Differentially expressed' column indicates whether the transcript was DE at an adjusted $p$-value of 0.1 in any of the DE analyses performed. The DE analyses were; R35 pre-embryo vs. post-embryo, lop138 pre-pollination vs. post-pollination, lop138 vs. R35 and lop138 vs. A36. More detail on the DE analyses can be found in Table 1 
identified (Additional file 1: Table S7). The transcripts identified as potential markers for stages undergoing embryo development in R35, lop138 and A36 encoded transcription factors known to function during seed development in Arabidopsis, such as MADS-box transcription factor PHERES1, Agamous-like MADS-box protein AGL61 and Nuclear transcription factor Y subunit B-6 and B-9 (Additional file 1: Table S8). Additional file 1: Table S9 lists potential markers of apomictic R35, i.e. transcripts active in one or more developmental stage of R35 but not active in one or both stage of lop 138 .

\section{Discussion}

Hieracium subgenus Pilosella contains both apomictic and sexual species, which have been developed into model systems for studying molecular and genetic components of apomixis [9]. Previous work used the aposporous $H$. praealtum (R35) for a deletion-based discovery of the two independent dominant loci conferring the key components of apomixes [16]. The locus LOSS OF APOMEIOSIS (LOA) was found to be responsible for avoidance of meiosis during embryo sac development and mutants missing this locus reverted to forming meiotically reduced embryo sacs. The locus LOSS OF PARTHENOGENESIS (LOP) was found necessary for parthenogenesis and autonomous endosperm development and mutants missing this locus reverted to the requirement of fertilization for embryo and endosperm development [16]. The availability of deletion mutants that reverted to the sexual mode of reproduction within the apomictic R35 background makes R35 and derived deletion mutants attractive for transcriptome studies. To facilitate discovery of transcriptional differences between apomixis and amphimixis, this study set the goal of producing a reproductive tissue reference transcriptome for subgenus Pilosella. R35, lop mutant accession (lop138) and an additional aposporous species belonging to subgenus Pilosella, $H$. auranticacum (A36), were used to produce the reference transcriptome.
To produce the transcriptome, we first needed to develop protocols for sampling reproductive tissue of Hieracium that can yield good quality RNA for sequencing. Here, a manual dissection procedure was developed which involved the use of RNase-free water for submerging florets during dissection and the use of an ammonium salt solution for storing collected ovules until RNA could be extracted. This procedure is novel in Hieracium and provides a valuable template for further work involving Hieracium and other Asteraceae species. The procedure for manually dissecting whole ovaries was relatively quick, thus ovaries were directly collected into RNA extraction buffer then processed to obtain high quality RNA from ovaries and ovules of Hieracium (Additional file 1: Table S1). Rabiger et al. [51] also reported manually dissecting Hieracium ovaries into liquid nitrogen then extracting RNA, which confirms the relative ease of sampling ovaries.

Developmental stages encompassing aposporous embryo sacs to autonomously developing seeds were sampled from R35 and A36. Developmental stages prior to pollination and post pollination were sampled from the mutant lop138. A reference transcriptome from all of these developmental stages was generated. This reference transcriptome is the first de novo transcriptome for Hieracium that combines data from all key events of apomixis (aposporous embryo sac development, parthenogenesis and autonomous endosperm developments) and partially sexual events (i.e. pollination-induced embryo and endosperm developments). The reference transcriptome has been deposited at the Shotgun Assembly project DDBJ/EMBL/ GenBank under the accession GEEH00000000.

The transcriptome was annotated through extensive BLAST searches and GO term mapping. Recently, ovary transcriptomes from two early apomictic events of $H$. praealtum (MMC and Functional Megaspore development stages) have been released by [51]. The reference transcriptome presented here provides additional

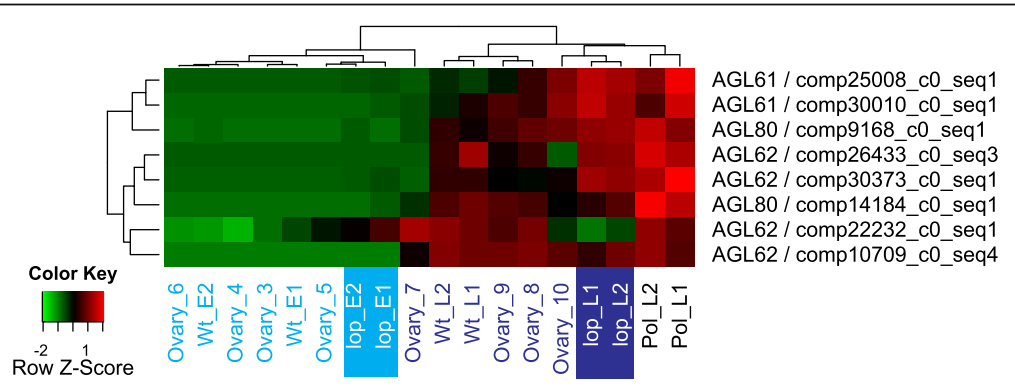

Fig. 6 Expression patterns of DE transcripts homologous to AGL61, AGL62 and AGL80. Hierarchically clustered expression heatmap with rows represent each transcript (labeled by the putative protein and the transcript ID) and relative expression (low in green and high in red) in the samples as indicated. Pre-embryo stages are indicated in light blue and post-embryo stages in dark blue text; the lop mutant pre-pollination (lop E1, E2) and post pollination (Iop L1 and L2) are also highlighted) 
resources for early apomictic events and is relevant to the key apomictic events of parthenogenesis and autonomous endosperm development, which makes it a valuable contribution to apomixis research. The representation of data from various stages and plant accessions (apomictic R35 and A36, and partially sexual lop138) makes the transcriptome useful for comparative analysis of apomictic and sexual Hieracium.

Differential expression analyses were performed to discover functional enrichment in the differentially expressed sequences of the apomictic and lop138 accessions. This was accomplished by identifying gene expression changes in response to pollination-independent seed development in R35 and the changes occurring in response to pollination-induced seed development in the mutant accession lop138 (Additional files 2, 3, 4, 5). Pollination-induced seed development in lop138 resulted in more transcriptional changes than pollination-independent seed development in R35. However, GO term enrichment analysis of the differentially expressed transcripts for R35 and lop 138 respectively, revealed that the two differentially expressed groups of transcripts shared many enriched GO term categories. There were few GO term categories enriched in lop 138 only, one of which was epigenetic regulation of gene expression. The other GO categories enriched in lop138 only were behavior, cell cycle and DNA metabolic process. Samples of lop138 were also compared to samples of the apomictic R35 and A36 accessions in order to discover enriched functions associated with differences between lop138 and the apomictic R35 and A36 (Additional files 4 and 5). Transcripts differentially expressed in lop 138 when compared to the apomictic accessions, resulted in enrichment of many GO term categories that were also shared by the classes of transcripts differentially expressed during seed development in lop138 and in R35 (Additional file 1: Figure S5). This highlights that apomictic and sexual seed developmental pathway display many common features. Many studies have suggested that deregulation of the sexual reproduction pathway is the likely cause of apomixis, hence apomixis and sexual reproduction are expected to share key components $[15,24]$.

Orthologs of molecular signals necessary for sexual reproduction, such as $E C 1$ and $R C D 1$ were identified as differentially expressed during fertilization-induced seed development in lop138 and their expression was also detected in R35 and A36 (Fig. 3). In Arabidopsis, EC1 proteins regulate gamete interaction during double fertilization, and are specifically expressed in the egg cell prior to fertilization [29]. In Hieracium, most of the putative EC1 orthologs were up-regulated in the pre-pollination sample of lop138, which suggests that in Hieracium these sequences may also have signaling roles prior to fertilization (Table 3). However, these sequences were also detected in apomictic R35 and A36, and although not identified as differentially expressed, most of the sequences showed expression in pre-embryo and post-embryo stages of R35 (Table 3). Similarly, orthologs of $R C D 1$, which is involved in the onset of sexual reproduction through its differentiation controlling function in yeast [28], were observed to be up-regulated during seed development in lop138, R35 and A36. The expression patterns observed from $E C 1$ and $R C D 1$ orthologs during this study suggest that molecular signals marking sexual reproduction can be detected in

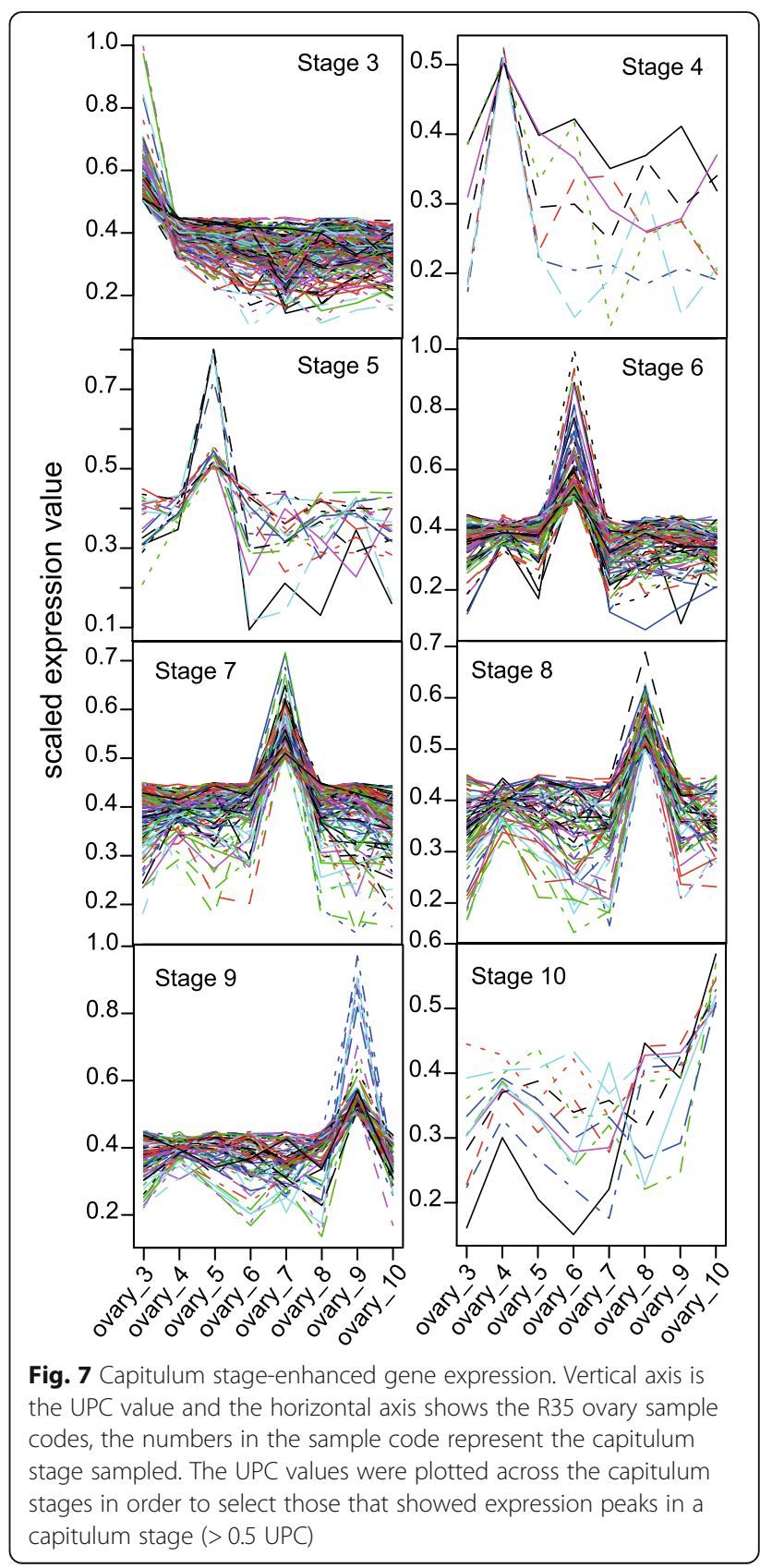


apomictic ovules and that apomictic seed development might be using elements of the sexual pathway to promote autonomous seed development. This corresponds with the current understanding that the apomixis pathway is overlaid on the default sexual pathway and uses elements of sexual reproduction to initiate apospory and autonomous seed development in somatic cells [24].

Koltunow et al. [24] were able to demonstrate that meiosis in the MMC, which is an initiation of the sexual pathway, was necessary for initiation of the AIs, which are the origins of aposporous embryo sacs. They performed experiments that specifically hindered meiosis in the MMC and observed that AIs and the subsequent aposporous embryo sacs were not being formed, which suggested that the function of $L O A$ was dependent on the initiation of the sexual pathway [24]. The $L O P$ locus may be operating in a similar manner to the $L O A$ locus in that it functions in response to cues of the sexual pathway. The identification of Hieracium sequences that are orthologous to genes involved in the initiation of sexual reproduction in other species, such as EC1 and $R C D 1$, is an important contribution towards understanding mechanisms of parthenogenesis in Hieracium.

Koltunow et al. [24] proposed that the two loci conferring apomixis in Hieracium, later determined to be $L O A$ and $L O P$, silence sexuality epigenetically or genetically. A similar model was proposed for the apomixis-controlling region (ACR) in Paspalum. The ACR was characterized by high cytosine methylation and was located on heterochromatin knobs [52]. Podio et al. [52] hypothesized that the ACR contained parthenogenesis activator and suppressor genes and that cytosine methylation in ACR could be inactivating the parthenogenesis suppressor genes, allowing precocious parthenogenesis to occur. To test their hypothesis, Podio et al. [52] specifically inhibited DNA methyltransferases, in order to prevent methylation, and discovered that parthenogenesis was negatively affected. The effect of demethylation in Paspalum is similar to the effect of deletion in the LOP locus in Hieracium, as LOP loss-of-function mutants revert to sexuality [16]. This suggests that genetic components that are either involved in suppressing sexuality or in suppressing parthenogenesis-suppressor genes were lost in lop mutants.

In addition to epigenetic regulation, pathways involved in biotic and abiotic signaling are likely to be involved in the induction of autonomous seed development. In R35, transcripts putatively encoding proteins with roles in auxin transport, response to stress, stem cell niche specification and maintenance and embryo patterning were up-regulated in pre-embryo and post-embryo stages of R35 (Fig. 5). In lop138, these pathways were up-regulated post-pollination (Fig. 5). These observations indicated that apomictic seed development might be induced through the effects of many pathways involving environmental cues, such as transport of the growth hormone auxin, response to stress and signal transduction. These pathways likely recruit genes involved in cell fate determination during early ovule development, resulting in precocious embryogenesis. Other studies also linked auxin to somatic embryogenesis [53] and to autonomous seed coat development in addition to endosperm initiation post-pollination [54]. Stress was also proposed to induce somatic embryogenesis [55] and many studies observed a relationship between stress-inducing signaling pathways and somatic embryogenesis induction [56-58].

The transcriptome data was also used to identify transcripts marking stages undergoing aposporous embryo sac development and embryo development. By utilizing abundance estimates for the transcriptome, transcripts preferentially active during aposporous embryo sac development in R35, during stages with developing embryos in R35, A36 and lop 138 were identified (Fig. 7; Additional file 10). These sequences can serve as a short list for investigating the developmental stages they mark. The validity of the list of developmental marker genes is demonstrated by the representation of genes already known to be associated with the respective developmental events, such as LOX2, AGL61 and PHE1. We identified an ortholog of $L O X 2$ as marking stages undergoing aposporous embryo sac development (Additional file 10 A), which is in agreement with previous studies that found LOX2 to be associated with AI cell. The same ortholog was down-regulated in mutants with deletion in $L O A$, i.e. the locus responsible for $\mathrm{AI}$ initiation $[51,59]$. AGL61, which is expressed specifically in the central cell of the female gametophyte and early endosperm in Arabidopsis [48, 60], was identified as a marker of stages undergoing embryo and endosperm development in the apomictic and the mutant accessions alike (Fig. 6). Similarly PHE1, which is a paternally expressed imprinted gene that is expressed in the embryo and endosperm of Arabidopsis [61, 62], was identified as marking stages undergoing embryo and endosperm development in the apomictic and the mutant accessions (Additional file $10 \mathrm{~B}$ ).

Seed development in R35 and A36 is largely autonomous (no paternal input), thus expression of PHE1, which is supposed to be silenced when inherited maternally and expressed when inherited paternally in Arabidopsis $[61,62]$ suggests a modified imprinting system in R35 and A36. [63], suggested that in pseudogamous apomicts, where the canonical $2 \mathrm{~m}: 1 \mathrm{p}$ genomic ratio of the endosperm is disrupted due to fertilization of two unreduced polar nuclei by a reduced sperm (4 m:1p genomic ratio), modification of the imprinting system could counteract the excess maternal genome in the 
endosperm. This could be achieved by reduced silencing of imprinted genes in the polar nuclei (paternalizing), thus increasing the dosage of paternally expressed imprinted genes [63]. Taken together, the identification of a transcriptionally active ortholog of a paternally expressed imprinted gene, PHE1, in both sexually derived seeds and apomictic seeds of Hieracium highlights that the imprinting system may be modified in Hieracium. Alternatively, imprinted genes of Hieracium may differ from those of Arabidopsis. Recent studies suggested that some imprinted genes might be species specific due to neofunctionalization of duplicated genes. For instance the FIS2-PRC2 complex is proposed to be Brasicaceae-specific and resulted from duplication of the VRN-PRC2 complex members that, in Arabidopsis, assumed a new role in gametophyte and seed development [64]. Genome duplication is a feature of the polyploid Hieracium species used in this study, therefore neofunctionalized imprinted genes could differ from those known in other species such as Arabidopsis. To simplify the analysis, duplicated transcripts were removed from the transcriptome, however, none of these transcripts were from PRC2 complex genes or genes involved in imprinting (Additional file 11).

The reference transcriptome is expected to be useful to further investigate the roles of PcG genes in Hieracium. Down-regulating members of the PcG complex that function to repress central cell proliferation prior to fertilization in Arabidopsis, such as FIE, did not result in autonomous endosperm development in sexual Hieracium $[22,23]$. FIE was not linked to the LOP locus in $H$. praealtum [24]. However, it was still needed for autonomous seed development as its down-regulation in apomictic Hieracium species resulted in decreased initiation of autonomous seed development and increased seed abortion [23]. In this study, only one sequence encoding a protein with similarity to Arabidopsis FIE (AtFIE) was expressed in all stages of R35 (Table 4). This agrees with a previous observation that a single allele of FIE was expressed in ovaries of apomictic and sexual Hieracium [23]. Although FIE was necessary for seed development in both apomictic and sexual Hieracium, it did not interact with many members of the PcG complex, such as MEA, MSI1 and RBR in vitro [23]. Hieracium proteins interacting with FIE are still unknown. [23] suggested SWINGER (EZI1) is likely to interact with FIE in Hieracium (HFIE), because it is a SET-domain containing PcG protein and shows higher homology to AtCLF than AtMEA. SWINGER's homology to AtCLF is important as AtCLF was the only PcG protein HFIE interacted with in vitro [23]. Here, sequences encoding proteins with high similarity to SWINGER and AtCLF were identified (Table 4). These sequences provide resources for future studies investigating the role of FIE during seed development in Hieracium.

\section{Conclusions}

Developing apomictic crop plants has the potential to revolutionise modern agriculture. It will provide a simple means to fix hybrid vigour by allowing clonal seed production from the high yielding individuals produced after crossing [2]. Farmers will then be able to collect and grow seed for the next season, enable efficient and consistent yields of high-quality seeds, fruits and vegetables at lower costs.

Our ultimate goal is to identify the genes conferring apomixis so that the trait can be engineered into crop species. To achieve this, we have been studying the model apomict Hieracium and have previously identified two loci (LOP and LOA) required for Hieracium to be an apomict. Mutant in the lop locus exhibits loss of parthenogenesis and autonomous endosperm development. The comprehensive floral reference transcriptome for Hieracium produced here provides a valuable resource for research into the molecular basis of apomixis and the identification of the genes underlying the LOP locus. Our results support the idea that apomictic and sexual seed developmental pathway share common components and that it is the switching on these pathways without the need for pollination that allows apomixis $[15,24]$. We identify many genes that play important roles in reproductive development and potential marker genes for different stages of seed development. These genes provide a way to examine in more fine detail the transcriptional changes associated with the key apomictic events of apomeiosis, parthenogenesis and autonomous endosperm development.

In R35, parthenogenesis and autonomous endosperm development are controlled by the $L O P$ locus and are inherited as a single unit. However, recombination is suppressed at the $L O P$ locus, which made resolving the level of dependency between the genetic components of parthenogenesis and autonomous endosperm formation in R35 difficult [16]. A study involving other apomictic and sexual species of Hieracium showed that autonomous endosperm could be genetically separated from parthenogenesis, suggesting parthenogenesis and autonomous endosperm formation might be controlled by separate components of the $L O P$ locus that are tightly linked in R35 [65]. The transcriptomic data produced for this study should contribute towards resolving the genetic components of the LOP locus. With the availability of gene models for Hieracium [51] and a substantial EST database and high-density genetic map of lettuce (Lactuca sativa) in Asteraceae [66], the RNA-seq data of R35 and its derivative mutant lop138 can be mapped to the genomic resources of Hieracium and 
lettuce. This transcriptome should help identify the genome-wide regulatory differences between the apomictic R35 and the mutant lop138 and the genetic components of the LOP loci involved in apomixis.

\section{Methods}

\section{Plant material}

Plant material was obtained from live specimens of wild type apomictic plants and a mutant plant ( $\Upsilon$ 138) derived from an apomictic accession [16]. The wild-type plant Hieracium praealtum (Vill.) Zahn is referred to by its accession code R35. For clarification, this plant was previously referred to as Hieracium caespitosum (C4D) [16] but has since been identified to be $H$. praealtum. Hieracium aurantiacum L. was used to pollinate the mutant accession and is referred by its accession code A36. The mutant accession Y138, referred to as 'lop138, was derived from R35 through gamma irradiation, which deleted the LOSS OF PARTHENOGENESIS (LOP) locus (Catanach, Erasmuson, Podivinsky, Jordan and Bicknell 2006). lop 138 retained the LOSS OF APOMEIOSIS $(L O A)$ locus, therefore it produces aposporous embryo sacs but fails to initiate autonomous seed development (Catanach, Erasmuson, Podivinsky, Jordan and Bicknell 2006). Upon fertilization lop 138 produces viable seeds and hybrid progeny with increased ploidy level compared with that of the maternal parent (Koltunow, Johnson, Rodrigues, et al. 2011). R35, lop138 and A36 were propagated in a heated glasshouse at the Department of Botany (University of Otago), in fertilized potting mix, under natural daylight augmented with SON-T AGRO 400w lights to provide $16 \mathrm{~h}$ of light per day.

\section{Screening embryo sacs for developing embryos}

To determine the stages of the capitulum that contained ovules undergoing embryo development, groups of florets were removed from capitula at the stages described by Koltunow et al. [9] and secured on a microscope slide with double-sided tape. A drop of water was then placed on the florets to submerge them and under a dissecting microscope the pappus of each floret was peeled away, along with the ovary walls. This exposed the ovule. A slit was made in the middle section of the ovule integuments with fine-tipped forceps (size 5 Dunmont forceps), which allowed the contents of the ovule to float in the surrounding water. The embryo sac could then be detached from the ovule with the forceps. Each embryo sac was washed off the forceps with safranin staining solution onto a new microscope slide. This was done under a microscope to ensure that the embryo sac was dislodged. The embryo sac was then left in the staining solution for $5 \mathrm{~min}$. After staining, the embryo sac was viewed under a compound microscope to discern the presence of the embryo.

\section{Procedure for tissue collections and RNA isolation}

To collect ovaries at specific stages, a group of florets were removed from the appropriate stage of capitulum development with forceps and laid on a microscope slide. Ovaries were cut off with a clean RNase free razor blade then put into RNA extraction buffer. After collecting all ovaries from a capitulum, ovaries were homogenized for subsequent RNA extraction. This method was used for capitula from stages 5 to 10 of Koltunow et al. [9]. Florets of stages 3 and 4 were too small to grasp with forceps, therefore the bracts were removed from a capitulum. Then, while still attached to the receptacle, florets were put on a microscope slide. The vegetative parts of florets were cut off and removed from the slide. Ovaries were scraped off the receptacle and put into lysis buffer for RNA extraction. RNA was extracted with MagMAX ${ }^{\mathrm{m}}$-96 Total RNA Isolation Kit (Ambion), which included a DNase step.

To collect ovule at specific stages, a floret was removed from the appropriate staged capitulum with forceps and attached to a microscope slide with doublesided tape as described above for staging. Then RNase free water was put on the floret to fully submerge it. Under a dissecting microscope the pappus was peeled away, along with the ovary walls. This exposed the ovule, which was removed with forceps and transferred into a tube containing storage medium. Multiple ovule material was combined and total RNA was extracted with RNAqueous ${ }^{\ominus}$-Micro Kit (Ambion) which included a DNase step. The RNA samples and yields are summarized in (Additional file 1: Table S1).

\section{RNA sequencing}

At the New Zealand Genomics Facility (NZGL) (University of Otago), TruSeq RNA Sample Prep Kits were used to prepare cDNA libraries from total RNA extracted from ovule and ovary tissue of R35, lop 138 and A36 (Table 1). RNA integrity was checked with the Agilent bioanalyzer 2100 and only RNA samples with RIN value over 7 were used for the cDNA preparation. A total of 18 cDNA libraries were prepared, eight of which were from ovary samples of R35 (not replicated) and five that were from ovule samples of R35, lop 138 and A36 with biological replication. These libraries were sequenced on an Illumina HiSeq2000, via the paired end sequencing method (100 bp), across four sequencing lanes. Quality control of the sequence reads was performed using FastQC (version 0.10.1) [67].

\section{De novo transcriptome assembly procedure}

To determine the relative expression levels of specific genes across the range of developmental stages and genotypes sampled, we first produced a de novo assembled reference transcriptome using sequence data from 
our 18 cDNA libraries. To produce the reference transcriptome the following was performed: DynamicTrim was run on the FASTQ data with the ' $h$ 20' parameter which sets a quality score cutoff of 20 . LengthSort was run on the trimmed data with the ' -150 ' parameter to select reads of at least 50 bases. The first 4,000,000 reads of each of the trimmed and sorted data files were combined into two separate files (one for each read1 and read2 of read pairs) and the Trinity assembler (version r2011-08-20) [68] was run on the resulting combined files. Alignment and generation of sam files was done using bwa aln and bwa sampe (0.6.1-r104).

\section{Assessment of assembly validity}

The validity of the reference assembly was assessed using two methods:

1. The percent of reads that mapped back to the reference assembly (PMBR) was calculated for each library. This was done by extracting and counting mapped reads of each alignment file (.sam) using samtools and the bash command wc - l. Total sequenced reads were also similarly counted from each .sam file. The PMBR was then calculated as (mapped reads / total reads)*100.

2. All available expressed sequence tag (EST) sequences for Hieracium and other Asteraceae species were downloaded from NCBI (last accessed on 4th April 2014). Standalone BLAST (ncbi-blast2.2.27+) [69] was used to perform BLASTN search of the newly reconstructed Hieracium transcriptome against these ESTs at an e-value cut-off of 1e-4.

\section{Assessment of completeness}

The completeness of the transcriptome was assessed based on sequence contiguity (N50), which is the minimum assembly size in which at least $50 \%$ of the assembled bases are found, and by searching for Benchmarking Universal Single-copy Orthologs (BUSCOs), in the Eukaryotic set of BUSCOs downloaded from http:// busco.ezlab.org/, and by searching the Hieracium transcriptome by using BUSCO_v1.1 software [70].

\section{Removal of chimera and redundant transcripts}

A total of $1,165,822$ plant protein reference sequences (RefSeq) were downloaded from NCBI (ftp://ftp.ncbi.nlm.nih.gov/refseq/release/) and used as a database for BLASTX (ncbi-blast-2.2.27+) [69] with an e-value cut-off of 1e-2. To remove chimeric transcripts, a pipeline and scripts by Yang and Smith [71] were obtained (https://bitbucket.org/yangya/optimize_assembler) and implemented. The results from the homology search were used to identify and remove chimeras from the transcriptome. To remove redundant transcripts, CD-HITS-EST version 4.5.4 $[72,73]$ was used to remove sequences that were entirely aligned to longer transcripts with a similarity threshold of $90 \%$. The redundant transcripts that were differentially expressed but removed are provided in Additional file 11.

\section{Annotation}

The annotation strategies are summarized in (Additional file 1: Table S10). To annotate the non-redundant transcriptome, two high-quality protein sequence databases were downloaded and formatted as BLAST databases. The two databases used the reference sequence database (RefSeq) containing protein sequences from plant species (downloaded from (ftp://ftp.ncbi.nlm.nih.gov/refseq/ release/) and all protein sequences included in the Swiss-Prot section of UniProtKB (downloaded from http://www.uniprot.org/uniprot). BLAST analysis (ncbi-blast-2.2.27+) [69] was used to search the Hieracium transcripts in the two protein databases using the BLASTX algorithm at an e-value cutoff of 1e-4. The results from these searches were merged and consolidated to produce a final annotation for the transcriptome. If a Hieracium transcript got hits to entries from both RefSeq and Swiss-Prot, the Swiss-Prot result was chosen as Swiss-Prot is manually curated and often supported via experimental or literature evidence. To identify novel transcripts potentially encoding full-length peptides, transcripts without any BLAST hits to EST or protein databases, were screened for Open Reading Frames (ORFs) using the ORF-predictor server (http:// proteomics.ysu.edu/tools/OrfPredictor.html). The resulting predicted peptides were filtered, using custom python and R Scripts (Additional file 12) to only retain transcripts with predicted peptides that are at least 100 amino acids long.

\section{Gene ontology term mapping}

After annotating the transcriptome via BLASTX, IDs of loci encoding proteins that matched the predicted peptides encoded by the query sequence (subject IDs) were used to assign GO terms to the transcripts. To accomplish this, the subject IDs in the BLASTX result, which came from two different databases (Swiss-Prot and RefSeq), were first converted to entrez gene IDs using the following steps: 1 ) The Uniprot 'Ac IDs' were mapped to entrez gene IDs on the uniprot ID mapping service (http://www.uniprot.org/uploadlists/). 2) The RefSeq 'gi IDs' were first mapped to UniProtKB 'AC IDs' then the 'AC IDs' mapped to entrez gene IDs on the uniprot web site (http://www.uniprot.org/uploadlists/). Using a custom $\mathrm{R}$ script (File S1), GO terms were mapped to the gene IDs from an NCBI file (gene2go.gz) (ftp://ftp.ncbi.nlm.nih.gov/gene/DATA/, last accessed on 
19th March 2015), which contains a comprehensive list of gene IDs and their corresponding GO IDs (File S2).

\section{Quantifying and normalizing expression}

To estimate transcript abundance across the sampled conditions, the number of reads supporting each transcript was counted from the alignment file (.sam). The reads aligned to each transcript, with a minimum mapping quality (MAQ) of 1 , were counted using the sam2count.py script [74]. The digital count data was then normalized using two different methods:

1. The Transcripts Per Million (TPM) method of normalization [75] was applied to correct for transcript length and sequencing depth effects. TPM is a slight modification of the popular RPKM method and it was calculated using the formula of [75] in a custom R script as follows; the normalization factor ( $\mathrm{z}$ ) was calculated by dividing each transcript's read count (tc) by its length ( $\mathrm{tl}$ ) and summing these values for each library $(\mathrm{z}=\operatorname{sum}(\mathrm{tc} / \mathrm{t} \mathrm{l}))$. The TPM for each transcript was then calculated by multiplying its read count by one million and dividing it by the product of its length and $\mathrm{z}\left(\mathrm{TPM}=\left(\mathrm{tc} \mathrm{c}^{* 1} \mathrm{e} 6\right) /\left(\mathrm{z}^{*} \mathrm{t} \mathrm{l}\right)\right)$.

2. The Universal exPression Code (UPC) method of normalization [49] was also used to scale the digital counts into values indicating the activity levels of genes from zero to one. The UPC package (version 2.10.8) was obtained from bioconductor (http:// bioconductor.org) and the 'UPC_RNASeq' function was implemented with user defined "GC annotation" enabled. A custom python script (Additional file 12) was used to count GC content of each transcript, which was then used as "GC annotation" file in UPC_RNASeq.

Figures 2, 3, 4, 5 and 6 were created using TPM values that were median centered and hierarchically clustered using heatmap. 2 function in R. For visualizing the expression of each transcript across the samples, the median centered and clustered expression values were scaled by $\mathrm{z}$-score and attributed a color, with bright red representing highest expression and light green representing lowest expression.

\section{Additional files}

Additional file 1: Additional Figures S1-S5 and Tables S1-S10. (DOCX $6791 \mathrm{~kb}$ )

Additional file 2: Differential expression analysis to discover changes associated with parthenogenesis in R35. (XLSX 256 kb)

Additional file 3: Differential expression analysis to discover changes associated with apomictic seed development in R35. (XLSX 1028 kb)
Additional file 4: Differential expression analysis to discover changes associated with sexually-induced seed development in lop138. (XLSX 2077 kb)

Additional file 5: Differential expression analysis to discover differences associated with quiescent embryo sac in lop138 relative to R35. (XLSX 2718 kb)

Additional file 6: GO term enrichment analysis in the differentially expressed transcripts resulting from comparing samples of apomictic accessions (R35 and A36) to samples of the mutant accession (lop138). (XLSX $4571 \mathrm{~kb}$ )

Additional file 7: Differentially expressed transcripts with no GO annotations and with or without BLAST hits. (XLSX $974 \mathrm{~kb}$ )

Additional file 8: Table of differentially expressed transcripts associated with "epigenetic regulation of gene expression". (XLSX 15 kb)

Additional file 9: Transcripts showing stage-preferred expression. (XLSX $61 \mathrm{~kb}$ )

Additional file 10: List of genes marking stages undergoing aposporous embryo sac development in R35. (XLSX 17 kb)

Additional file 11: Transcripts that are differentially expressed but identified as duplicates and removed from transcriptome during processing. (XLSX $68 \mathrm{~kb}$ )

Additional file 12: $\mathrm{R}$ scripts. (DOCX $20 \mathrm{~kb}$ )

\section{Acknowledgements}

We would like to thank Mik Black, Benoit Auvray, Peter Stockwell and Rudiger Brauning for helping with the bioinformatics and statistical analyses; Rob Day and Robyn Lee for guidance with tissue and RNA isolation; and Susan Mackenzie for help growing the plants used in this study.

\section{Funding}

This work was supported by the University of Otago, through a Postgraduate Scholarship to SB and Priming Partnerships funding.

\section{Availability of data and materials}

The datasets generated and analysed during the current study are available in the Sequence Read Archive at https://www.ncbi.nlm.nih.gov/ under the bioproject number 'PRJNA284533'. The reference transcriptome is deposited at the Shotgun Assembly project DDBJ/EMBL/GenBank under the accession number GEEH00000000.

\section{Authors' contributions}

$\mathrm{SB}, \mathrm{AC}, J \mathrm{~L}, \mathrm{RB}$, and $\mathrm{RM}$ designed the experiments. SB performed the experiments. SB, AC, RB and RM analysed the data. SB and RM wrote and AC, $J \mathrm{~L}$ and $\mathrm{RB}$ edited the manuscript. All authors read and approved the final manuscript.

Ethics approval and consent to participate

Not applicable.

\section{Consent for publication}

Not applicable.

\section{Competing interests}

The authors declare that they have no competing interests.

\section{Publisher's Note}

Springer Nature remains neutral with regard to jurisdictional claims in published maps and institutional affiliations.

\section{Author details}

'Department of Biochemistry, University of Otago, 710 Cumberland St, Dunedin 9016, New Zealand. ²Department of Botany, University of Otago, 464 Great King St, Dunedin 9016, New Zealand. ${ }^{3}$ New Zealand Institute for Plant and Food Research, Gerald St, Lincoln 7608, New Zealand. 
Received: 20 May 2018 Accepted: 10 September 2018 Published online: 24 September 2018

\section{References}

1. Nogler GA. Gametophytic Apomixis. In: Johri PBM, editor. Embryology of Angiosperms: 1st edn. Berlin: Springer; 1984. p. 475-518.

2. Spillane C, Curtis MD, Grossniklaus U. Apomixis technology developmentvirgin births in farmers' fields? Nat Biotechnol. 2004;22(6):687-91.

3. Savidan Y. Chromosomal and embryological analyses in sexual $x$ apomictic hybrids of Panicum maximum Jacq. TAG Theor Appl Genet. 1980;58(2):153-6.

4. Roche D, Cong P, Chen Z, Hanna WW, Gustine DL, Sherwood RT, OziasAkins P. Short communication: an apospory-specific genomic region is conserved between Buffelgrass (Cenchrus ciliaris L.) and Pennisetum squamulatum Fresen. Plant J. 1999;19(2):203-8.

5. Pessino SC, Ortiz JPA, Leblanc O, do Valle CB, Evans C, Hayward MD. Identification of a maize linkage group related to apomixis in Brachiaria. Theor Appl Genet. 1997;94(3):439-44.

6. Naumova TN, Osadtchiy JV, Sharma VK, Dijkhuis P, Ramulu KS. Apomixis in plants: structural and functional aspects of diplospory in Poa nemoralis and P. palustris. Protoplasm 1999;208(1-4):186-95.

7. Albertini E, Marconi G, Barcaccia G, Raggi L, Falcinelli M. Isolation of candidate genes for apomixis in Poa pratensis L. Plant Mol Biol. 2004;56(6):879-94.

8. Noyes RD, Rieseberg LH. Two independent loci control agamospermy (Apomixis) in the triploid flowering plant Erigeron annuus. Genetics. 2000;155(1):379-90.

9. Koltunow AM, Johnson SD, Bicknell RA. Sexual and apomictic development in Hieracium. Sex Plant Reprod. 1998;11(4):213-30.

10. Matzk F, Prodanovic S, Baumlein H, Schubert I. The inheritance of apomixis in Poa pratensis confirms a five locus model with differences in gene expressivity and penetrance. Plant Cell. 2005;17(1):13-24.

11. Hörandl E. The evolution of self-fertility in apomictic plants. Sex Plant Reprod. 2010;23(1):73-86.

12. Koltunow AMG, Johnson SD, Okada T. Apomixis in hawkweed: Mendel's experimental nemesis. J Exp Bot. 2011;62(5):1699-707.

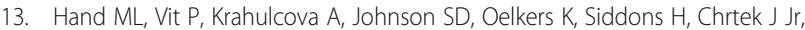
Fehrer J, Koltunow AM. Evolution of apomixis loci in Pilosella and Hieracium (Asteraceae) inferred from the conservation of apomixis-linked markers in natural and experimental populations. Heredity. 2015;114(1):17-26.

14. Bicknell RA, Koltunow AM. Understanding apomixis: recent advances and remaining conundrums. Plant Cell. 2004;16:S228-45.

15. Tucker MR, Araujo AC, Paech NA, Hecht V, Schmidt ED, Rossell JB, De Vries SC, Koltunow AM. Sexual and apomictic reproduction in Hieracium subgenus pilosella are closely interrelated developmental pathways. Plant Cell. 2003;15(7):1524-37.

16. Catanach AS, Erasmuson SK, Podivinsky E, Jordan BR, Bicknell R. Deletion mapping of genetic regions associated with apomixis in Hieracium. Proc Natl Acad Sci U S A. 2006;103(49):18650-5.

17. Polegri L, Calderini O, Arcioni S, Pupilli F. Specific expression of apomixislinked alleles revealed by comparative transcriptomic analysis of sexual and apomictic Paspalum simplex Morong flowers. J Exp Bot. 2010;61(6):1869-83.

18. Gutiérrez-Marcos JF, Costa LM, Prà MD, Scholten S, Kranz E, Perez P, Dickinson HG. Epigenetic asymmetry of imprinted genes in plant gametes. Nat Genet. 2006;38(8):876-8.

19. Gehring M, Bubb KL, Henikoff S. Extensive demethylation of repetitive elements during seed development underlies gene imprinting. Science. 2009;324(5933):1447-51.

20. Hsieh T-F, Ibarra CA, Silva P, Zemach A, Eshed-Williams L, Fischer RL, Zilberman D. Genome-wide demethylation of Arabidopsis endosperm. Science. 2009;324(5933):1451-4

21. Zemach A, Kim MY, Silva P, Rodrigues JA, Dotson B, Brooks MD, Zilberman D. Local DNA hypomethylation activates genes in rice endosperm. Proc Natl Acad Sci U S A. 2010;107(43):18729-34.

22. Rodrigues JCM, Luo M, Berger F, Koltunow AMG. Polycomb group gene function in sexual and asexual seed development in angiosperms. Sex Plant Reprod. 2009;23(2):123-33.

23. Rodrigues JCM, Tucker MR, Johnson SD, Hrmova M, Koltunow AMG. Sexual and apomictic seed formation in Hieracium requires the plant polycombgroup gene FERTILIZATION INDEPENDENT ENDOSPERM. Plant Cell. 2008;20(9):2372-86.
24. Koltunow AM, Johnson SD, Rodrigues JC, Okada T, Hu Y, Tsuchiya T, Wilson $\mathrm{S}$, Fletcher $\mathrm{P}$, Ito K, Suzuki G, et al. Sexual reproduction is the default mode in apomictic Hieracium subgenus Pilosella, in which two dominant loci function to enable apomixis. Plant J. 2011;66(5):890-902.

25. Byzova MV, Franken J, Aarts MGM, Almeida-Engler J, Engler G, Mariani C, Campagne MMVL, Angenent GC. Arabidopsis STERILE APETALA, a multifunctional gene regulating inflorescence, flower, and ovule development. Genes Dev. 1999;13(8):1002-14.

26. Lei M. The MCM complex: its role in DNA replication and implications for cancer therapy. Curr Cancer Drug Targets. 2005;5(5):365-80.

27. Longhese MP, Plevani P, Lucchini G. Replication factor a is required in vivo for DNA replication, repair, and recombination. Mol Cell Biol. 1994; 14(12):7884-90.

28. Okazaki N, Okazaki K, Watanabe Y, Kato-Hayashi M, Yamamoto M, Okayama $\mathrm{H}$. Novel factor highly conserved among eukaryotes controls sexual development in fission yeast. Mol Cell Biol. 1998;18(2):887-95.

29. Sprunck S, Rademacher S, Vogler F, Gheyselinck J, Grossniklaus U, Dresselhaus T. Egg cell-secreted EC1 triggers sperm cell activation during double fertilization. Science. 2012;338(6110):1093-7.

30. Okamoto K, Ueda H, Shimada T, Tamura K, Koumoto Y, Tasaka M, Morita MT Hara-Nishimura I. An ABC transporter B family protein, ABCB19, is required for cytoplasmic streaming and gravitropism of the inflorescence stems. Plant Signal Behav. 2016;11(3):e1010947.

31. Shigeto J, Tsutsumi Y. Diverse functions and reactions of class III peroxidases. New Phytol. 2016;209:1395-402.

32. Shahollari B, Vadassery J, Varma A, Oelmüller R. A leucine-rich repeat protein is required for growth promotion and enhanced seed production mediated by the endophytic fungus Piriformospora indica in Arabidopsis thaliana. Plant J. 2007:50(1):1-13.

33. Jun JH, Ha CM, Fletcher JC. BLADE-ON-PETIOLE1 coordinates organ determinacy and axial polarity in Arabidopsis by directly activating ASYMMETRIC LEAVES2. Plant Cell. 2010;22(1):62-76.

34. Ha CM, Jun JH, Fletcher JC. Control of Arabidopsis leaf morphogenesis through regulation of the YABBY and KNOX families of transcription factors. Genetics. 2010;186(1):197-206.

35. Bell E, Creelman RA, Mullet JE. A chloroplast lipoxygenase is required for wound-induced jasmonic acid accumulation in Arabidopsis. Proc Natl Acad Sci U S A. 1995;92(19):8675-9.

36. Bannenberg G, Martínez M, Hamberg M, Castresana C. Diversity of the enzymatic activity in the lipoxygenase gene family of Arabidopsis thaliana. Lipids. 2009;44(2):85-95.

37. Santos MO, Aragão FJL. Role of SERK genes in plant environmental response. Plant Signal Behav. 2009;4(12):1111-3.

38. Geldner N, Richter S, Vieten A, Marquardt S, Torres-Ruiz RA, Mayer U, Jürgens $G$. Partial loss-of-function alleles reveal a role for GNOM in auxin transport-related, post-embryonic development of Arabidopsis. Development. 2004;131(2):389-400.

39. Dhonukshe P, Huang F, Galvan-Ampudia CS, Mähönen AP, Kleine-Vehn J, Xu J, Quint A, Prasad K, Friml J, Scheres B, et al. Plasma membranebound AGC3 kinases phosphorylate PIN auxin carriers at TPRXS(N/S) motifs to direct apical PIN recycling. Development. 2010;137(19): 3245-55.

40. Bennett $T$, Sieberer $T$, Willett $B$, Booker J, Luschnig $C$, Leyser $O$. The Arabidopsis MAX pathway controls shoot branching by regulating Auxin transport. Curr Biol. 2006;16(6):553-63.

41. Laux T, Mayer KF, Berger J, Jürgens G. The WUSCHEL gene is required for shoot and floral meristem integrity in Arabidopsis. Development. 1996; 122(1):87-96.

42. Stahl Y, Simon R. Plant stem cell niches. Int J Dev Biol. 2005;49(5-6):479-89.

43. Wu X, Dabi T, Weigel D. Requirement of homeobox gene STIMPY/WOX9 for Arabidopsis meristem growth and maintenance. Curr Biol. 2005;15(5): 436-40.

44. Di Laurenzio L, Wysocka-Diller J, Malamy JE, Pysh L, Helariutta Y, Freshour G, Hahn MG, Feldmann KA, Benfey PN. The SCARECROW gene regulates an Asymmetric cell division that is essential for generating the radial Organization of the Arabidopsis Root. Cell. 1996;86(3):423-33.

45. Jenik PD, Gillmor CS, Lukowitz W. Embryonic patterning in Arabidopsis thaliana. Annu Rev Cell Dev Biol. 2007;23(1):207-36.

46. Portereiko MF, Lloyd A, Steffen JG, Punwani JA, Otsuga D, Drews GN. AGL80 is required for central cell and endosperm development in Arabidopsis. Plant Cell. 2006;18(8):1862-72. 
47. Kang I-H, Steffen JG, Portereiko MF, Lloyd A, Drews GN. The AGL62 MADS domain protein regulates cellularization during endosperm development in Arabidopsis. Plant Cell. 2008;20(3):635-47.

48. Steffen JG, Kang I-H, Portereiko MF, Lloyd A, Drews GN. AGL61 interacts with AGL80 and is required for central cell development in Arabidopsis. Plant Physiol. 2008;148(1):259-68.

49. Piccolo SR, Withers MR, Francis OE, Bild AH, Johnson WE. Multiplatform single-sample estimates of transcriptional activation. Proc Natl Acad Sci U S A. 2013;110(44):17778-83.

50. Xie C, Mao X, Huang J, Ding Y, Wu J, Dong S, Kong L, Gao G, Li C-Y, Wei L. KOBAS 2.0: a web server for annotation and identification of enriched pathways and diseases. Nucleic Acids Res. 2011;39(suppl 2):W316-22.

51. Rabiger DS, Taylor JM, Spriggs A, Hand ML, Henderson ST, Johnson SD, Oelkers K, Hrmova M, Saito K, Suzuki G, et al. Generation of an integrated Hieracium genomic and transcriptomic resource enables exploration of small RNA pathways during apomixis initiation. BMC Biol. 2016;14:86

52. Podio M, Cáceres ME, Samoluk SS, Seijo JG, Pessino SC, Ortiz JPA, Pupilli F. A methylation status analysis of the apomixis-specific region in Paspalum spp. suggests an epigenetic control of parthenogenesis. J Exp Bot. 2014;65(22): 6411-24.

53. Su YH, Zhao XY, Liu YB, Zhang CL, O'Neill SD, Zhang XS. Auxin-induced WUS expression is essential for embryonic stem cell renewal during somatic embryogenesis in Arabidopsis. Plant J. 2009;59(3):448-60.

54. Figueiredo DD, Batista RA, Roszak PJ, Hennig L, Köhler C. Auxin production in the endosperm drives seed coat development in Arabidopsis. Elife. 2016; 5:e20542.

55. Yang $X$, Zhang X. Regulation of somatic embryogenesis in higher plants. Crit Rev Plant Sci. 2010;29(1):36-57.

56. Jin F, Hu L, Yuan D, Xu J, Gao W, He L, Yang X, Zhang X. Comparative transcriptome analysis between somatic embryos (SEs) and zygotic embryos in cotton: evidence for stress response functions in SE development. Plant Biotechnol J. 2014;12(2):161-73.

57. Salvo SAGD, Hirsch CN, Buell CR, Kaeppler SM, Kaeppler HF. Whole Transcriptome profiling of maize during early somatic embryogenesis reveals altered expression of stress factors and embryogenesis-related genes. PLoS One. 2014;9(10):e111407.

58. Wickramasuriya AM, Dunwell JM. Global scale transcriptome analysis of Arabidopsis embryogenesis in vitro. BMC Genomics. 2015;16:301.

59. Okada T, Hu Y, Tucker MR, Taylor JM, Johnson SD, Spriggs A, Tsuchiya T, Oelkers K, Rodrigues JCM, Koltunow AMG. Enlarging cells initiating apomixis in Hieracium praealtum transition to an embryo sac program prior to entering mitosis. Plant Physiol. 2013;163(1):216-31.

60. Bemer M, Wolters-Arts M, Grossniklaus U, Angenent GC. The MADS domain protein DIANA acts together with AGAMOUS-LIKE80 to specify the central cell in Arabidopsis ovules. Plant Cell. 2008;20(8):2088-101.

61. Köhler C, Page DR, Gagliardini V, Grossniklaus U. The Arabidopsis thaliana MEDEA Polycomb group protein controls expression of PHERES1 by parental imprinting. Nat Genet. 2005;37(1):28-30.

62. Köhler C, Hennig L, Spillane C, Pien S, Gruissem W, Grossniklaus U. The Polycomb-group protein MEDEA regulates seed development by controlling expression of the MADS-box gene PHERES1. Genes Dev. 2003; 17(12):1540-53.

63. Spielman M, Vinkenoog R, Scott RJ. Genetic mechanisms of apomixis. Philos Trans R Soc Lond Ser B Biol Sci. 2003;358(1434):1095-103.

64. Qiu Y, Liu S-L, Adams KL. Concerted divergence after gene duplication in Polycomb repressive complexes. Plant Physiol. 2017;174(2):1192-204.

65. Ogawa D, Johnson SD, Henderson ST, Koltunow AMG. Genetic separation of autonomous endosperm formation (AutE) from the two other components of apomixis in Hieracium. Plant Reproduction. 2013;26(2):113-23.

66. Truco MJ, Ashrafi H, Kozik A, Leeuwen H, Bowers J, Reyes Chin Wo S, Stoffel K, Xu H, Hill T, Van Deynze A, et al. An ultra-high-density, transcript-based, genetic map of lettuce. G3 (Bethesda). 2013;3(4):617-31.

67. Andrews S: A quality control tool for high throughput sequence data. 2010. http://www.bioinformatics.babraham.ac.uk/projects/fastqc/

68. Grabherr MG, Haas BJ, Yassour M, Levin JZ, Thompson DA, Amit I, Adiconis X, Fan L, Raychowdhury R, Zeng Q, et al. Trinity: reconstructing a full-length transcriptome without a genome from RNA-Seq data. Nat Biotechnol. 2011; 29(7):644-52.

69. Altschul SF, Gish W, Miller W, Myers EW, Lipman DJ. Basic local alignment search tool. J Mol Biol. 1990;215(3):403-10.
70. Simao FA, Waterhouse RM, loannidis P, Kriventseva EV, Zdobnov EM. BUSCO: assessing genome assembly and annotation completeness with single-copy orthologs. Bioinformatics. 2015;31:3210-2.

71. Yang Y, Smith SA. Optimizing de novo assembly of short-read RNA-seq data for phylogenomics. BMC Genomics. 2013;14(1):328.

72. Li W, Jaroszewski L, Godzik A. Clustering of highly homologous sequences to reduce the size of large protein databases. Bioinformatics. 2001;17(3):282-3.

73. Li W, Godzik A. Cd-hit: a fast program for clustering and comparing large sets of protein or nucleotide sequences. Bioinformatics. 2006;22(13):1658-9.

74. Buffalo V. sam2counts.py - convert SAM mapping results to reference sequence counts. 2010. https://github.com/vsbuffalo/sam2counts.

75. Wagner GP, Kin K, Lynch VJ. Measurement of mRNA abundance using RNAseq data: RPKM measure is inconsistent among samples. Theory Biosci. 2012;131(4):281-5.
Ready to submit your research? Choose BMC and benefit from:

- fast, convenient online submission

- thorough peer review by experienced researchers in your field

- rapid publication on acceptance

- support for research data, including large and complex data types

- gold Open Access which fosters wider collaboration and increased citations

- maximum visibility for your research: over $100 \mathrm{M}$ website views per year

At $\mathrm{BMC}$, research is always in progress.

Learn more biomedcentral.com/submissions 